\title{
Uneven Two-Sided Power Distributions: \\ With Applications in Econometric Models
}

Samuel Kotz ${ }^{1}$ and J. René van Dorp ${ }^{2}$

Engineering Management and Systems Engineering Department

The George Washington University, Washington D.C. 20052

dorpjr@gwu.edu; kotz@seas.gwu.edu

\begin{abstract}
In this paper we propose and analyze a bounded density function with a jump discontinuity at a threshold. Its properties are presented and a maximum likelihood estimation (MLE) procedure for the threshold location and jump size is developed. The distribution seems be appropriate in the context of financial engineering, production analysis, standard auction models and the equilibrium job search problem. An example of the MLE procedure is given utilizing an i.i.d. sample of standardized log differences of bi-monthly US Certificate Deposit interest rates for the period from 1966 - 2002. The corresponding time series was constructed using an Auto-Regressive Conditional Heteroscedastic (ARCH) model.
\end{abstract}

Keywords: CD Interest Rates, Maximum Likelihood Estimation, Discontinuities.

\section{Introduction}

The concept of symmetry permeates a multitude of phenomena in the physical world and plays an important role in numerous human activities, in particular in Arts and Sciences. The classical book by H. Weyl (1952) delineates numerous situations which involve symmetry. In architecture

\footnotetext{
${ }^{1}$ Professor and Research Scholar, Engineering Management and Systems Engineering Department, The George Washington University, 1776 G Street, NW, Suite 110, Washington D.C., 20052. E-mail: kotz@gwu.edu

${ }^{2}$ Same address. Associate Professor, Corresponding Author. E-mail: dorpjr@gwu.edu
} 
the ancient Greeks were the promoters of symmetry in their classical structures and monuments. In modern arts, the Dutch artist M.C. Escher (1889 - 1972) achieved striking effects in his work exploring mathematical symmetry (see, e.g., Escher 1989). The basic symmetry operations: reflection, rotation, double reflection and translation constitute the symmetry group for an object or a figure. It has direct applications in crystallography, amongst other fields. The distinction of symmetries with respect to a given point (center of symmetry), a line (axis of symmetry) and a plane (plane of symmetry) are also important for applications. Human beings and many animals have symmetric proportions. A line from a human's nose to the ground would divide him/her into equal symmetric parts - manifesting bilateral symmetry . The symmetry of a wheel generates radial symmetry, which is present in many statistical distributions including the basic multivariate Gaussian (or normal) distribution. For a more recent discussion on the topic of symmetry see Zabell (1988).

Figure 1A below depicts the symmetric two-parameter Gaussian distribution together with an empirical probability density function (pdf) of standardized (to ensure homoscedasticity) logdifferences of bi-monthly US certificate deposit (CD) rates. The time series of these standardized log differences were constructed utilizing the Auto-Regressive Conditional Heteroscedastic (ARCH) model due to Engle (1982) (see Section 5 for further details). Similarly to the analysis in Klein (1993) (who studied interest rate data on 30-year Treasury bonds from 1977 to 1990) Figure 1A shows that the empirical pdf of the financial data by far is too peaked to be captured by a normal pdf. Figure 1B displays the three-parameter asymmetric Laplace with the pdf

$$
f_{\theta, \kappa, \sigma}(x)= \begin{cases}\frac{\sqrt{2}}{\sigma} \frac{\kappa}{1+\kappa^{2}} \exp \left[-\sqrt{2} \frac{1}{\sigma \kappa}(\theta-x)\right], & \text { for } x<\theta \\ \frac{\sqrt{2}}{\sigma} \frac{\kappa}{1+\kappa^{2}} \exp \left[-\sqrt{2} \frac{\kappa}{\sigma}(x-\theta)\right], & \text { for } x \geq \theta\end{cases}
$$

$\sigma, \kappa>0$, suggested by Kozubowski and Podgórski (1999) to capture such a peak which seems to be a characteristic of financial data. Along these lines, Van Dorp and Kotz (2002a,b) proposed a bounded four-parameter Two-Sided Power (TSP) distribution (Figure 1C) 


$$
f(x \mid a, \theta, b, n)= \begin{cases}\frac{n}{(b-a)}\left(\frac{x-a}{\theta-a}\right)^{n-1}, & \text { for } a<x \leq \theta \\ \frac{n}{(b-a)}\left(\frac{b-x}{b-\theta}\right)^{n-1}, & \text { for } \theta<x<b\end{cases}
$$

with support $[a, b], a \leq \theta \leq b, n>0$ having an explicit line of symmetry at $\theta$ since the powers of the functional expression in both branches are both equal to $(n-1)$.

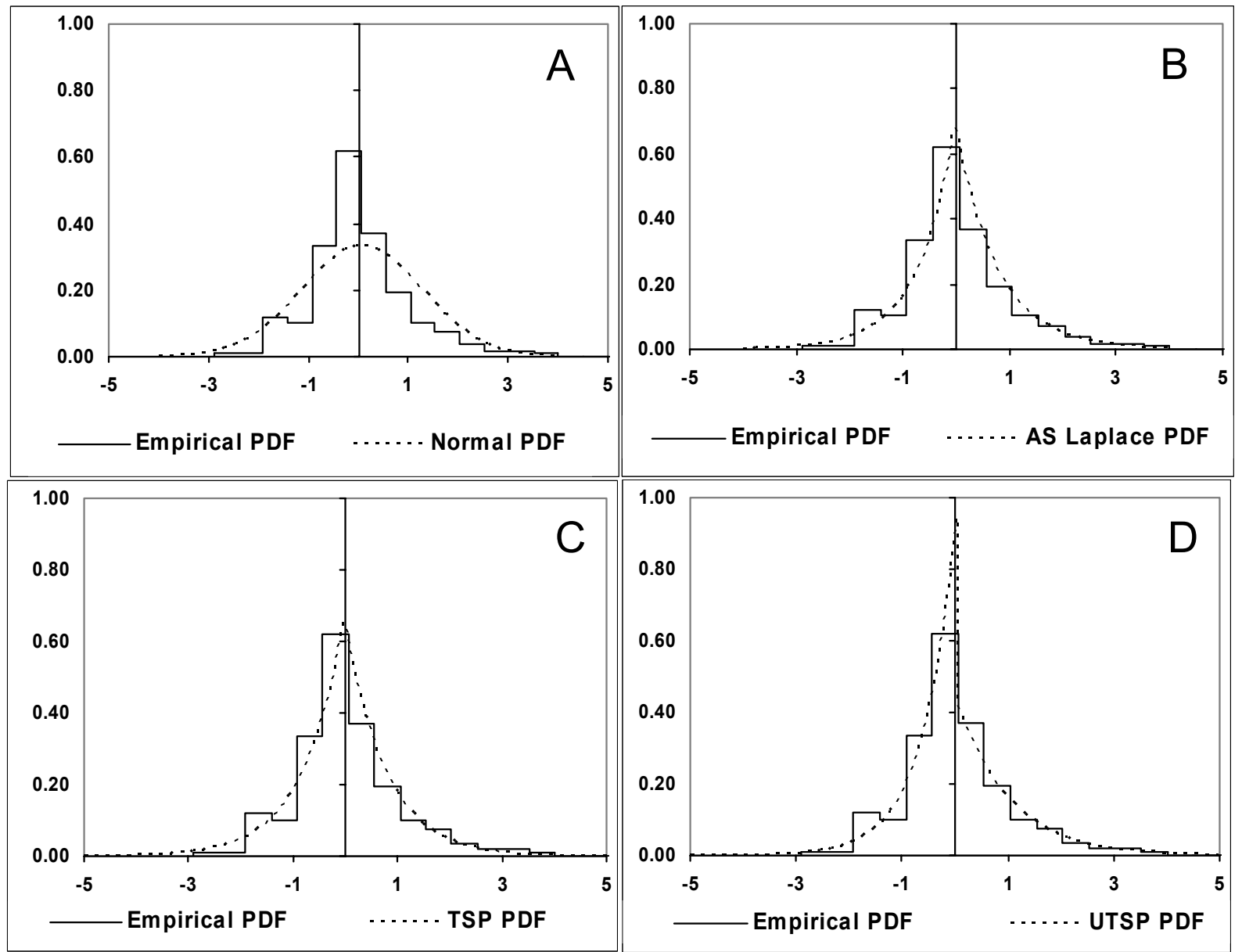

Fig. 1. Empirical PDF of two-step log differences and ML fitted distributions. A: Gaussian (or Normal); B:

Asymmetric Laplace; C: Two Sided Power (TSP) ; D: Uneven Two Sided Power (UTSP)

Finally, Figure 1D depicts an apparently novel asymmetric generalization of the TSP distribution that allows for unequal powers in the branches and a jump discontinuity at $\theta$. All four distributions in Figure 1 were fitted via MLE procedures. It will be shown herein that amongst 
the distributions in Figure 1, the asymmetric generalization of the TSP distribution, to be referred as Uneven TSP (UTSP) distribution, provides the "better fit" to the empirical pdf of standardized log differences of bi-monthly US certificate deposit (CD) rates from 1966-2002.

Modifications of the type shown in Figure 1D have been available for the Gaussian distributions for a long time, notably with applications in communication theory and signal detection (see, e.g., Fechner 1897; Kanefsky and Thomas 1965; Barnard 1989). Figure 2 displays an example of an asymmetric Gaussian distribution with a jump discontinuity analogous to the one in Figure 1D.

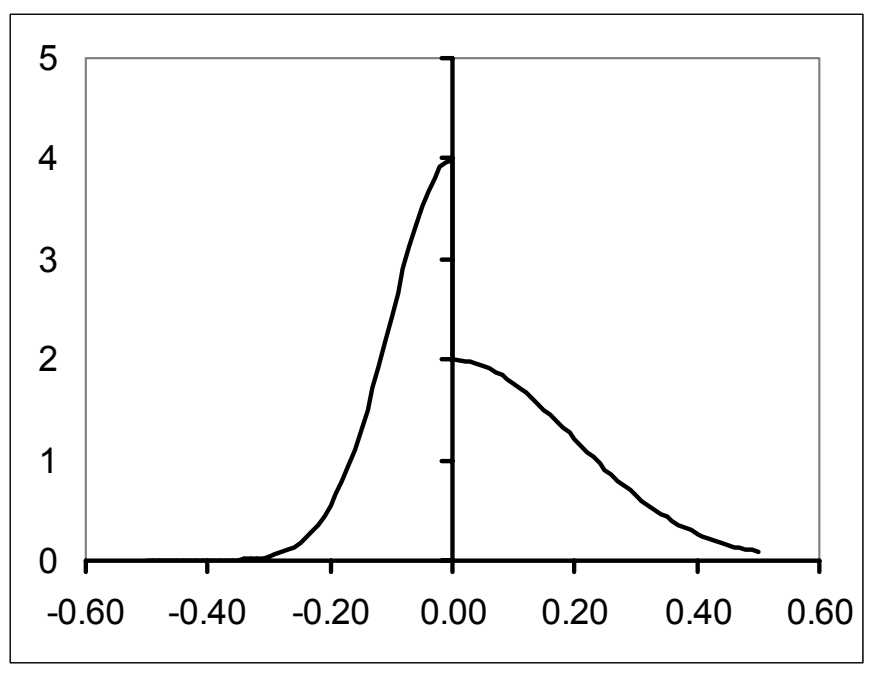

Fig. 2. Example of an Asymmetric Gaussian Distribution

Distributions of the type depicted in Figure 2 are also used with an increasing frequency in econometric applications as error terms in linear regression models. Aigner et al. (1976) were apparently the first to propose a model with a conditional density jump in the context of production analysis; more recent applications can be found, for example, in standard auction models and the equilibrium job search problems. In standard auction models (see, e.g., Donald and Paarsch 1996) the density jumps from zero to a positive value and in the equilibrium job search applications the density jumps from one level to another, inducing kinks in the cumulative 
distribution function (cdf) (see, e.g., Bowlus et al. 2001). Chernozhukov and Hong (2001) discuss recently the regression inference problem for the model originally suggested by Aigner et al. mentioned above.

It turns out that for the TSP distribution given by (2) an analogous structure can be obtained by appropriately manipulating the central part of the so-called generalized trapezoidal distributions (cf. Van Dorp and Kotz 2003a) which has originally been constructed to imitate three stage uncertainty phenomena that often occur in the physical world. In this context, trapezoidal distributions have been used in the screening and detection of cancer (see, e.g., Flehinger and Kimmel 1987; Brown 1999). By shrinking the central part of the generalized trapezoidal distribution to a single point we arrive at the UTSP distribution involving four parameters (in the case when the boundaries, determining the range, are assumed to be known). Note that the transition from the continuous generalized trapezoidal case to the discontinuous UTSP case can easily be achieved by just one single operation.

In Section 2, the density of the UTSP distribution will be derived. Some properties of UTSP distributions are discussed in Section 3. While in inference problems for asymmetric Gaussian distributions the threshold parameter is often assumed to be known, a maximum likelihood procedure is developed in Section 4 for UTSP distributions with unknown threshold parameter, unknown size of the jump discontinuity, as well as unknown powers in the respective branches of the pdf curve (but with known boundary parameters). In Section 5 the MLE procedure derived in Section 4 will be exemplified using standardized log differences of bi-monthly US certificate deposit (CD) rates for the period 1966 - 2002. We shall also compare the ML fitted UTSP distribution to ML fitted Gaussian, asymmetric Laplace and TSP distributions presented in Figure 1. Details of derivations for the MLE procedure for UTSP distributions are presented in the Appendix. 


\section{Uneven two-sided power distribution}

Trapezoidal distributions have been advocated in risk analysis problems by Pouliquen (1970) and more recently by Powell and Wilson (1997), among others. These distributions have also found application as membership functions in fuzzy set theory (see, e.g., Chen and Hwang 1992). However, trapezoidal distributions consisting of three stages are somewhat restrictive, since the growth and decay (in the first and third stages) are limited in this case to linear functions while the middle stage represents complete (flat) stability rather than a possible (mild) incline or decline (see Figure 3A). The trapezoidal probability density function (depicted in Figure $3 \mathrm{~A}$ ) is of the form

$$
f_{X}(x \mid a, b, c, d)=\frac{2}{d+c-b-a} g(x \mid a, b, c, d)
$$

where $a \leq b \leq c \leq d$ and the function

$$
g(x \mid a, b, c, d)= \begin{cases}\frac{x-a}{b-a}, & \text { for } a \leq x<b \\ 1, & \text { for } b \leq x<c \\ \frac{d-x}{d-c}, & \text { for } c \leq x<d \\ 0, & \text { elsewhere }\end{cases}
$$
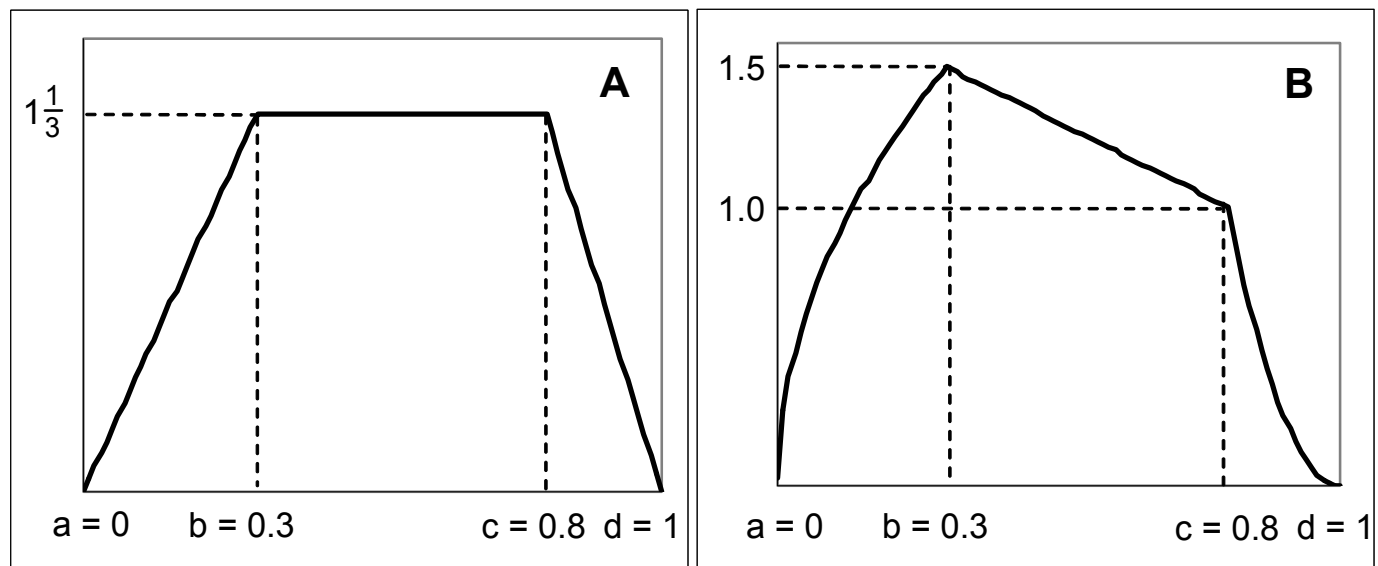

Fig. 3. A: A Trapezoidal distribution with parameters $a=0, b=0.3, c=0.8$ and $d=1$.

B: A Generalized Trapezoidal Distribution with parameters

$$
a=0, b=0.3, c=0.8, d=1, n_{1}=1.5, n_{3}=3, \text { and } \alpha=1.5 .
$$


Generalized trapezoidal distributions (see Van Dorp and Kotz 2003a) inherit the four basic trapezoidal parameters $a, b, c$ and $d$, and require, for its complete description, two additional parameters $n_{1}$ and $n_{3}$ specifying the growth and decay rates at the first and third stages of the distribution respectively, and the boundary ratio parameter $\alpha>0$ satisfying

$$
f_{X}(b)=\alpha f_{X}(c) .
$$

The density function of the generalized trapezoidal distribution is then given by

$$
\begin{gathered}
f_{X}\left(x \mid a, b, c, d, n_{1}, n_{3}, \alpha\right)= \\
\begin{cases}\frac{2 \alpha n_{1} n_{3}}{2 \alpha(b-a) n_{3}+(\alpha+1)(c-b) n_{1} n_{3}+2(d-c) n_{1}}\left(\frac{x-a}{b-a}\right)^{n_{1}-1}, & \text { for } a \leq x<b \\
\frac{2 n_{1} n_{3}}{2 \alpha(b-a) n_{3}+(\alpha+1)(c-b) n_{1} n_{3}+2(d-c) n_{1}}\left\{(\alpha-1) \frac{c-x}{c-b}+1\right\}, & \text { for } b \leq x<c \\
\frac{2 n_{1} n_{3}}{2 \alpha(b-a) n_{3}+(\alpha+1)(c-b) n_{1} n_{3}+2(d-c) n_{1}}\left(\frac{d-x}{d-c}\right)^{n_{3}-1}, & \text { for } c \leq x<d \\
0, & \text { elsewhere }\end{cases}
\end{gathered}
$$

where $a<b<c<d, n_{1}>0, n_{3}>0$ and $\alpha>0$. Here the growth and decay may exhibit a nonlinear convex or concave behavior and $f_{X}(b)$ and $f_{X}(c)$ do not necessarily take the same value (cf. (5)). Figure 3B provides a graph of the pdf of a standard generalized trapezoidal distribution with support $[0,1]$.

Expression(6) for the generalized trapezoidal density is obtained (see, e.g., Van Dorp and Kotz 2003a) using a mixture of the three densities $f_{X_{1}}, f_{X_{2}}, f_{X_{3}}$

$$
f_{X}(x)= \begin{cases}\sum_{i=1}^{3} \pi_{i} f_{X_{i}}(x), & \text { for } a \leq x<d \\ 0, & \text { elsewhere }\end{cases}
$$

where $\sum_{i=1}^{3} \pi_{i}=1, \pi_{i}>0$ and

$$
\begin{gathered}
f_{X_{1}}\left(x \mid a, b, n_{1}\right)=\left(\frac{n_{1}}{b-a}\right)\left(\frac{x-a}{b-a}\right)^{n_{1}-1}, \quad a \leq x<b, n_{1}>0, \\
f_{X_{2}}(x \mid b, c, \alpha)=\frac{2}{(\alpha+1)(c-b)^{2}}\{(1-\alpha) x+\alpha c-b\}, \quad b \leq x \leq c, \alpha>0,
\end{gathered}
$$




$$
f_{X_{3}}\left(x \mid c, d, n_{3}\right)=\left(\frac{n_{3}}{d-c}\right)\left(\frac{d-x}{d-c}\right)^{n_{3}-1}, \quad c \leq x<d, n_{3}>0
$$

the mixture probabilities being

$$
\left\{\begin{array}{l}
\pi_{1}=\frac{2 \alpha(b-a) n_{3}}{2 \alpha(b-a) n_{3}+(\alpha+1)(c-b) n_{1} n_{3}+2(d-c) n_{1}} \\
\pi_{2}=\frac{(\alpha+1)(c-b) n_{1} n_{3}}{2 \alpha(b-a) n_{3}+(\alpha+1)(c-b) n_{1} n_{3}+2(d-c) n_{1}} \\
\pi_{3}=\frac{2(d-c) n_{1}}{2 \alpha(b-a) n_{3}+(\alpha+1)(c-b) n_{1} n_{3}+2(d-c) n_{1}}
\end{array}\right.
$$

By collapsing the central part in (6) to a single point we arrive at the UTSP distribution involving 4 parameters (the boundaries are assumed to be known). Specifically, letting $c \downarrow b$ we have

$$
\left\{\begin{aligned}
\pi_{1} & \rightarrow \frac{\alpha(b-a) n_{3}}{\alpha(b-a) n_{3}+(d-b) n_{1}} \\
\pi_{2} & \rightarrow 0 \\
\pi_{3} & \rightarrow \frac{(d-b) n_{1}}{\alpha(b-a) n_{3}+(d-b) n_{1}}
\end{aligned}\right.
$$

Note that here $\pi_{1}+\pi_{3}=1$. Hence, letting $c \downarrow b$ yields the UTSP distribution with the pdf

$$
f_{X}\left(x \mid a, b, d, n_{1}, n_{3}, \alpha\right)=\left\{\begin{array}{cl}
\frac{\alpha n_{1} n_{3}}{\alpha(b-a) n_{3}+(d-b) n_{1}}\left(\frac{x-a}{b-a}\right)^{n_{1}-1}, & \text { for } a \leq x<b \\
\frac{n_{1} n_{3}}{\alpha(b-a) n_{3}+(d-b) n_{1}}\left(\frac{d-x}{d-b}\right)^{n_{3}-1}, & \text { for } b \leq x<d
\end{array}\right.
$$

with support $[a, d]$ and the cdf

$$
F_{X}\left(x \mid a, b, d, n_{1}, n_{3}, \alpha\right)= \begin{cases}0, & \text { for } x<a \\ \frac{\alpha(b-a) n_{3}}{\alpha(b-a) n_{3}+(d-b) n_{1}}\left(\frac{x-a}{b-a}\right)^{n_{1}}, & \text { for } a \leq x<b \\ 1-\frac{(d-b) n_{1}}{\alpha(b-a) n_{3}+(d-b) n_{1}}\left(\frac{d-x}{d-b}\right)^{n_{3}}, & \text { for } b \leq x<d \\ 1, & \text { for } x>d .\end{cases}
$$

Figure 4 displays the resulting UTSP distribution generated from the generalized trapezoidal distribution in Figure 3B. Substituting $\alpha=1$ and $n_{1}=n_{3}=n$ into (13) we arrive at the pdf of the TSP distribution given by (2). Note that, the distribution (2) can be also be obtained directly by generalizing the triangular family of distributions (see, e.g., Van Dorp and Kotz 2002a). 


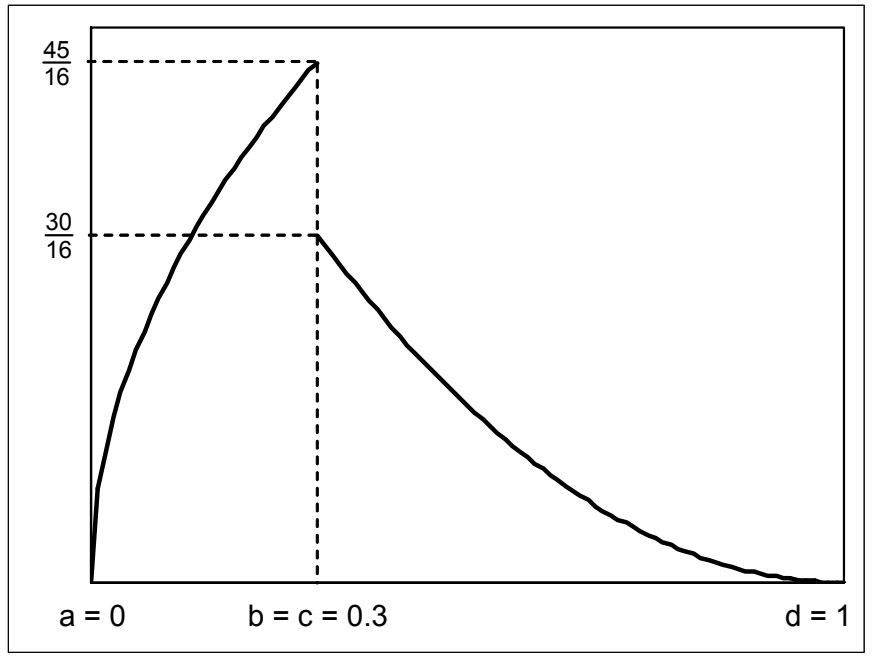

Fig. 4. Example of an Uneven Two-sided Power Distribution with parameters

$$
a=0, b=0.3, d=1, n_{1}=1.5, n_{3}=3 \text { and } \alpha=1.5 .
$$

\section{Some properties of the uneven STSP distribution}

In deriving the properties we shall restrict ourselves to Uneven STSP (USTSP) distributions with the support $[0,1]$ by setting $a=0, d=1$ and $b=\theta(0 \leq \theta \leq 1)$ in (13). This yields the density

$$
f_{X}(x \mid \Theta)= \begin{cases}p \frac{n_{1}}{\theta}\left(\frac{x}{\theta}\right)^{n_{1}-1}, & \text { for } 0 \leq x<\theta \\ (1-p) \frac{n_{3}}{1-\theta}\left(\frac{1-x}{1-\theta}\right)^{n_{3}-1}, & \text { for } \theta \leq x \leq 1\end{cases}
$$

where the single mixing probability $p$ is given by

$$
p=\frac{\alpha \theta n_{3}}{\alpha \theta n_{3}+(1-\theta) n_{1}}=\frac{\theta \alpha n_{3}}{\theta\left(\alpha n_{3}-n_{1}\right)+n_{1}} .
$$

Denote

$$
\Theta=\left\{\theta, n_{1}, n_{3}, \alpha\right\},
$$

to be the vector of the four parameters, where $0 \leq \theta \leq 1, n_{1}>0, n_{3}>0, \alpha>0$ (Compare with the more general expressions (12) for $p$ and the pdf (13) for the standardized density (15)) . 
Properties for the general case (13) follow directly from those obtained in the standardized case (15) by utilizing a simple scale transformation. The possible geometrical shapes of the USTSP pdf given by (15) are similar to those of the STSP distribution (or of the beta distribution, see Van Dorp and Kotz 2002a) including the J-shaped and U-shaped forms and in addition allow for a jump discontinuity at $\theta$.

Setting $\alpha=1$ in (15) and (16) yields a continuous generalization of the Standard Two-Sided Power (STSP) distribution (with two parameters)

$$
f_{X}(x \mid \theta, n)= \begin{cases}n\left(\frac{x}{\theta}\right)^{n-1}, & \text { for } 0 \leq x<\theta \\ n\left(\frac{1-x}{1-\theta}\right)^{n-1}, & \text { for } \theta \leq x \leq 1\end{cases}
$$

(compare with the four-parameter density (2)) with the pdf

$$
f_{X}\left(x \mid \theta, n_{1}, n_{3}\right)= \begin{cases}\frac{n_{1} n_{3}}{\theta n_{3}+(1-\theta) n_{1}}\left(\frac{x}{\theta}\right)^{n_{1}-1}, & \text { for } 0 \leq x<\theta \\ \frac{n_{1} n_{3}}{\theta n_{3}+(1-\theta) n_{1}}\left(\frac{1-x}{1-\theta}\right)^{n_{3}-1}, & \text { for } \theta \leq x \leq 1,\end{cases}
$$

by allowing for different powers $n_{1}$ and $n_{3}$ in the two respective branches of the STSP density given by (18). We shall refer to the densities given by (19) as Generalized STSP (GSTSP) distributions. In the limiting cases $\theta=1$ and $\theta=0,(15)$ simplifies to a power distribution or its reflection, respectively. We thus have in the increasing order of complexity: Triangular distributions (cf. (2) with $n=2$ ), Trapezoidal distributions (cf. (3)), Standard TSP distributions (STSP, cf. (18)), four-parameter TSP distributions (cf. (2)), Generalized Standard TSP distributions (GSTSP, cf. (19)), Generalized TSP distributions (GTSP, cf. (13) with $\alpha=1$ ), Uneven Standard TSP distributions (USTSP cf. (15)), the six-parameter Uneven TSP distributions (cf. (13)) and the seven-parameter Generalized Trapezoidal Distributions (cf. (6)). The reader is advised to produce a table of these nine distributions and the corresponding pdf graph. 
The cdf associated with the density (15) is continuous but non-differentiable at the "threshold" $\theta$ and is given by

$$
F_{X}(x \mid \Theta)= \begin{cases}0, & \text { for } x \leq 0 \\ p\left(\frac{x}{\theta}\right)^{n_{1}}, & \text { for } 0<x<\theta \\ 1-(1-p)\left(\frac{1-x}{1-\theta}\right)^{n_{3}-1}, & \text { for } \theta \leq x<1 \\ 1, & \text { for } x \geq 1\end{cases}
$$

From (20) (the cdf of the USTSP case) we obtain that

$$
F_{X}(\theta \mid \Theta)=p
$$

Hence, the total probability mass is split into two parts $p$ and $(1-p)($ cf. (16)) at $\theta$. For the STSP distribution (cf. (18)) expression (21) simplifies to $F_{X}(\theta \mid \Theta)=\theta$ regardless of the value of $n$. The latter property is referred to as the "hinge" property of the STSP family (see, e.g., Van Dorp and Kotz 2002a).

From (15) and (5) we have

$$
\frac{f_{X}^{-}(\theta \mid \Theta)}{f_{X}^{+}(\theta \mid \Theta)}=\frac{\lim _{x} f_{X}(x \mid \Theta)}{\lim _{x \downarrow \theta} f_{X}(x \mid \Theta)}=\frac{p \frac{n_{1}}{\theta}}{(1-p) \frac{n_{3}}{1-\theta}}=\alpha .
$$

While in case of a generalized trapezoidal distribution $\alpha$ was referred to as a boundary ratio parameter, for the USTSP distribution $\alpha$ could be interpreted as a jump parameter. In case $\alpha=1$, there is no jump at the threshold parameter $\theta$, in case $\alpha>1(\alpha<1)$ the density jumps down (up) at $\theta$, with larger (smaller) values of the density indicating a larger jump down (up). The size of the jump discontinuity at the threshold parameter $\theta$ may be derived utilizing (22) and the definition of $p(16)$ to be

$$
\left|f_{X}^{-}(\theta \mid \Theta)-f_{X}^{+}(\theta \mid \Theta)\right|=\frac{|1-\alpha| \alpha n_{1} n_{3}}{\alpha \theta n_{3}+(1-\theta) n_{1}} .
$$

(Recall that for USTSP distributions $a=0, d=1$ and $b=\theta$ ). 
Moments of USTSP distributions (15) follow immediately using the (inherited) mixture structure (7), the mixing weight $p(16)$ and the moments of a (one-sided) power distribution on $[0, \theta]$ and its reflection on $[\theta, 1]$, yielding

$$
E\left[X^{k} \mid \Theta\right]=p \frac{n_{1} \theta^{k}}{n_{1}+k}+(1-p)\left[n_{3} \sum_{i=0}^{k}\left(\begin{array}{c}
k \\
i
\end{array}\right)(-1)^{i} \frac{(1-\theta)^{i}}{n_{3}+i}\right], k=1,2, \ldots
$$

Consequently

$$
E[X \mid \Theta]=p\left[\frac{n_{1} \theta}{n_{1}+1}\right]+(1-p)\left[\frac{n_{3} \theta+1}{n_{3}+1}\right]
$$

and

$$
E\left[X^{2} \mid \Theta\right]=p\left[\frac{n_{1}\left(n_{1}+1\right) \theta^{2}}{\left(n_{1}+2\right)\left(n_{1}+1\right)}\right]+(1-p)\left[\frac{2+2 n_{3} \theta+n_{3}\left(n_{3}+1\right) \theta^{2}}{\left(n_{3}+2\right)\left(n_{3}+1\right)}\right]
$$

Substituting for $p$ as given by (16) into (25) and (26) we arrive at the expressions for the first two moments in terms of the four parameters $n_{1}, n_{3}, \theta$ and $\alpha$, respectively. A derivation of a closed form expression for the variance $\left(\sigma^{2}\right)$ is somewhat tedious, but straightforwardly follows utilizing (16), (25) and (26) and the definition

$$
\sigma^{2}=E\left[X^{2} \mid \Theta\right]-E^{2}[X \mid \Theta]
$$

Using modern computational facilities one may easily calculate higher moments of USTSP distribution from (24) including skewness and kurtosis, which definitely may be of practical interest.

The behavior of the mixing probability $p$ given by (16) as a function of the jump parameter $\alpha$ and of the threshold parameter $\theta$ may be of interest (recall that the jumps in the value of the density occur at $\theta$ ). Figure 5 displays the mixture probability $p$ as function of $\theta$ for five different values of $\alpha$ from 0.1 up to 10 for the case when $n_{1}=n_{3}=2$. Setting $\alpha=1, n_{1}=n_{3}=2$ in (16) and (15) results in the well known triangular distribution - in this case $p=\theta-$ with the property that the probability mass to the left of the mode equals the distance of the mode to the lower bound relative to the range of the whole support. This property is preserved by the TSP 
generalization (2) of the triangular distribution and follows by substituting $\alpha=1, n_{1}=n_{3}=n$ into (16). From Figure 5 we conclude that in the USTSP distribution case with $n_{1}=n_{3}=n$ (or equivalently, $n_{1} / n_{3}=1$ ) the probability mass to the left of the threshold parameter is less (larger) than its relative distance from the lower bound when the density jumps up, i.e. $\alpha<1$, (down, i.e. $\alpha>1$, ) at the threshold. Finally, when $\left(n_{1} / n_{3}\right)<1(>1)$ in a USTSP distribution (cf. (15) and (16)) an even smaller (larger) probability mass is assigned to the left of the threshold parameter than it is in the case $n_{1} / n_{3}=1$. The limiting behavior of the mixing probability $p$ as a function of one of the parameters $n_{1}, n_{3}, \alpha$ and $\theta$, while keeping the others fixed, follows directly from (16). In fact, $p \downarrow 0(p \uparrow 1)$ when $n_{1} \rightarrow \infty$, or $n_{3} \downarrow 0$, or $\alpha \downarrow 0$, or $\theta \downarrow 0$ (when $n_{1} \downarrow 0$, or $n_{3} \rightarrow \infty$, or $\alpha \rightarrow \infty$, or $\left.\theta \uparrow 1\right)$.

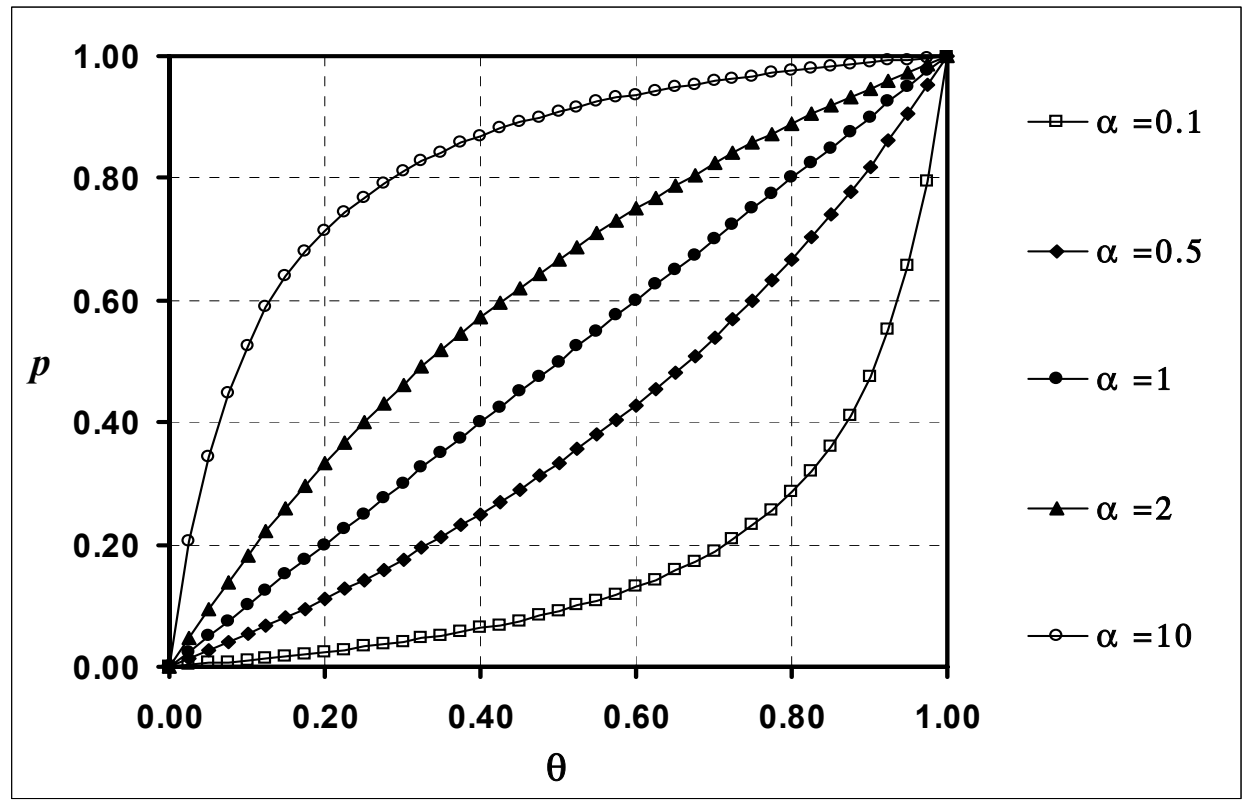

Fig. 5. Behavior of Mixture Probability $p$ as a function of the threshold parameter $\theta$ for different values of the jump parameter $\alpha$ for $n_{1}=n_{3}=2$.

The behavior of the mean $E[X \mid \Theta](25)$ as a function of the jump parameter $\alpha$ (while keeping the other parameters fixed) follows directly from (25) and (16). In fact, when $\alpha$ increases, the mixture probability $p$ increases, assigning a larger weight to the mean value of the power 
distribution on $[0, \theta]$ in (25) (and a smaller weight to the mean value of the reflected power distribution on $[\theta, 1])$ and hence results in a decrease of $E[X \mid \Theta]$. In a similar manner one can derive the behavior of $E[X \mid \Theta]$ as a function of the other parameters as well.

Table 1 summarizes the limiting behavior of the mixture probability $p(16)$, the limiting pdf of (15) with the mean $E[X \mid \Theta]$ (25) under a variety of limiting scenarios. All the parameters in the parameter vector $\Theta(17)$ that do not appear in the first column of Table 1 are assumed to be fixed.

Table 1. Limiting behavior of the mixture probability $p(16)$, the pdf (15) and the mean $E[X \mid \Theta](25)$ under a variety of scenarios. Parameters not mentioned in the first column are assumed to be fixed.

\begin{tabular}{ccccc}
\hline Row & Scenario & $p$ & $f_{X}\left(x \mid \theta, n_{1}, n_{3}\right)$ & $E[X \mid \Theta]$ \\
\hline 1. & $\alpha \rightarrow \infty$ & $\uparrow 1$ & $\rightarrow\left(\frac{x}{\theta}\right)^{n_{1}-1}$ on $[0, \theta]$ & $\downarrow \frac{n_{1} \theta}{n_{1}+1}$ \\
\hline 2. & $\theta \downarrow 0$ & $\uparrow 1$ & single point mass at 0 & $\downarrow 0$ \\
\hline 3. & $n_{1} \rightarrow \infty$ & $\uparrow 1$ & single point mass at $\theta$ & $\downarrow \theta$ \\
\hline 4. & $n_{3} \downarrow 0$ & $\uparrow 1$ & $\rightarrow\left(\frac{x}{\theta}\right)^{n_{1}-1}$ on $[0, \theta]$ & $\downarrow \frac{n_{1} \theta}{n_{1}+1}$ \\
\hline 5. & $\alpha \downarrow 0$ & $\downarrow 0$ & $\rightarrow\left(\frac{1-x}{1-\theta}\right)^{n_{3}-1}$ on $[\theta, 1]$ & $\uparrow \frac{n_{3} \theta+1}{n_{3}+1}$ \\
\hline 6. & $\theta \uparrow 1$ & $\downarrow 0$ & single point mass at 1 & $\uparrow 1$ \\
\hline 7. & $n_{1} \downarrow 0$ & $\downarrow 0$ & $\rightarrow\left(\frac{1-x}{1-\theta}\right)^{n_{3}-1}$ on $[\theta, 1]$ & $\uparrow \frac{n_{3} \theta+1}{n_{3}+1}$ \\
\hline 8. & $n_{3} \rightarrow \infty$ & $\downarrow 0$ & single point mass at $\theta$ & $\uparrow \theta$ \\
\hline 9. & $n_{1}, n_{3} \rightarrow \infty$, & $=\frac{\alpha \theta}{\alpha \theta+\beta(1-\theta)}$ & single point mass at $\theta$ & $\rightarrow \theta$ \\
& $\frac{n_{1}}{n_{3}}=\beta$ & \multicolumn{2}{c}{} & \\
\hline 10. & $n_{1}, n_{3} \downarrow 0$ & $=\frac{\alpha \theta}{\alpha \theta+\beta(1-\theta)}$ & Bernoulli $(p, 1-p)$ & $\rightarrow 1-p$ \\
& $\frac{n_{1}}{n_{3}}=\beta$ & at 0 and 1 & \\
\hline
\end{tabular}

Some brief comments on the indicative results presented in Table 1 are in order. The scenarios in Rows 2) $\theta \downarrow 0$; 3) $n_{1} \rightarrow \infty$; 6) $\theta \uparrow 1$ and 8) $n_{3} \rightarrow \infty$ all result in a single point limiting density at the values of $\theta$ (or specifically, limiting values 0 or 1 of $\theta$ in rows 2 and 6 , respectively). Also the last two limiting scenarios in Table 1 (the 9-th and 10-th row) keeping 
$n_{1} / n_{3}=\beta$ constant result in the same value of the mixture probability $p$ (since $p$ as given in (16) depends on the ratio $n_{1} / n_{3}$ ), but yield very different limiting distributions: a single point mass at $\theta$ when $n_{1}, n_{3} \rightarrow \infty$ and a two-point Bernoulli distribution (with parameter $p$ ) at 0 and 1 when $n_{1}, n_{3} \downarrow 0$. This is because when $n_{1}, n_{3} \downarrow 0$ the structure of the original pdf (15) becomes Ushaped with an anti-mode at $\theta$. The two situations in rows $1(\alpha \rightarrow \infty)$ and $4\left(n_{3} \downarrow 0\right)$ result in the same limiting density $(x / \theta)^{n_{1}}$ on $[0, \theta]$ and equivalently $\alpha \downarrow 0$ and $n_{1} \downarrow 0$ (in rows 5 and 7 ) both yield the density $\{(1-x) /(1-\theta)\}^{n_{3}-1}$ on $[\theta, 1]$.

\section{MLE procedure for USTSP distributions}

Here, we shall derive a maximum likelihood procedure for USTSP distributions that is algorithmically straightforward in terms of elementary function evaluations. Let for a sample of size $m$ with the values $\underline{X}=\left(X_{1}, \ldots, X_{m}\right)$ the order statistics be $X_{(1)}<X_{(2)}<\ldots<X_{(m)}$. Utilizing (15) and (16), the likelihood $\mathcal{L}(\underline{X} \mid \Theta)$ for $\underline{X}$ is, by definition,

$$
\prod_{i=1}^{r} \frac{\alpha n_{1} n_{3}}{\alpha \theta n_{3}+(1-\theta) n_{1}}\left(\frac{X_{(i)}}{\theta}\right)^{n_{1}-1} \prod_{i=r+1}^{m} \frac{n_{1} n_{3}}{\alpha \theta n_{3}+(1-\theta) n_{1}}\left(\frac{1-X_{(i)}}{1-\theta}\right)^{n_{3}-1}
$$

where $r$ is defined so that $X_{(r)} \leq \theta<X_{(r+1)}$, with $X_{(0)} \equiv 0, X_{(m+1)} \equiv 1$. Collecting the terms in (28) we obtain

$$
\begin{aligned}
& \mathcal{L}(\underline{X} \mid \Theta)= \\
& \left(n_{1} n_{3}\right)^{m}\left[\frac{\alpha^{r}}{\left\{\alpha \theta n_{3}+(1-\theta) n_{1}\right\}^{m}}\right]\left[\prod_{i=1}^{r} \frac{X_{(i)}}{\theta}\right]^{n_{1}-1}\left[\prod_{i=r+1}^{m} \frac{1-X_{(i)}}{1-\theta}\right]^{n_{3}-1} .
\end{aligned}
$$

The difficulty in maximizing (29) as a function of the parameters $n_{1}, n_{3}, \alpha$ and $\theta$ is due to an irregular behavior of $\mathcal{L}(\underline{X} \mid \Theta)$ as a function of the threshold parameter $\theta$. Figure 6 depicts an example of $\mathcal{L}(\underline{X} \mid \Theta)$ as function of the $\theta$ for the case $n_{1}=n_{3}=2$ and $\alpha=1$ and the eight $(m=8)$ order statistics

$$
\begin{aligned}
& X_{(1)}=0.10 ; X_{(2)}=0.25 ; X_{(3)}=0.30 ; X_{(4)}=0.40 ; \\
& X_{(4)}=0.45 ; X_{(6)}=0.60 ; X_{(7)}=0.75 ; X_{(8)}=0.80 .
\end{aligned}
$$


When $n_{1}=n_{3}=n$ and $\alpha=1$ in $(29), \mathcal{L}(\underline{X} \mid \Theta)$ is simplified to the likelihood associated with the STSP distribution given by (18). Van Dorp and Kotz (2002a) have shown that for the STSP distribution with $n \geq 1$ the maximum of $\mathcal{L}(\underline{X} \mid \Theta)$ as a function of $\theta$ is attained at one of the order statistics $X_{(i)}, i=1, \ldots, m$ (as it happens to be in Figure 6). This may not be the case for the more general likelihood given by (29). The following numerical algorithm to determine the maximum of (29) and hence the maximum likelihood estimators $\widehat{\alpha}, \widehat{n_{1}}, \widehat{n_{3}}$ and $\widehat{\theta}$ is proposed for the case $n_{1} \geq 1$ and $n_{3} \geq 1$. An empirical pdf of the data under consideration (see Figure 1) could confirm the precondition $n_{1} \geq 1$ and $n_{3} \geq 1$ of the numerical algorithm. The precondition $\left(n_{1} \geq 1, n_{3} \geq 1\right)$ for the ML procedure below can be relaxed to allow for the remaining parameter scenarios $\left(n_{1} \geq 1,0 \leq n_{3} \leq 1\right),\left(0 \leq n_{3} \leq 1, n_{3} \geq 1\right)$ and $\left(0 \leq n_{1} \leq 1,0 \leq n_{3} \leq 1\right)$ by appropriate modifications of the details regarding Step 4 (provided in the Appendix). The readers are encouraged to investigate these details on their own.

Iteration $k:(k=1,2, \ldots)$

(Don't confuse this ordinal $k$ with the order of the moments in (24))

Step 1: Given $\left(n_{3}\right)_{k}, \alpha_{k}$, and $\theta_{k}$ maximize $\mathcal{L}(\underline{X} \mid \Theta)$ for $\left(n_{1}\right)_{k+1}$

Step 2: Given $\left(n_{1}\right)_{k+1}, \alpha_{k}$, and $\theta_{k}$ maximize $\mathcal{L}(\underline{X} \mid \Theta)$ for $\left(n_{3}\right)_{k+1}$

Step 3: Given $\left(n_{1}\right)_{k+1}$ and $\left(n_{3}\right)_{k+1}$ and $\theta_{k}$ maximize $\mathcal{L}(\underline{X} \mid \Theta)$ for $\alpha_{k+1}$

Step 4: Given $\left(n_{1}\right)_{k+1}$ and $\left(n_{3}\right)_{k+1}$ and $\alpha_{k+1}$ maximize $\mathcal{L}(\underline{X} \mid \Theta)$ for $\theta_{k+1}$

Step 5: Go back to Step 1, unless the pre-assigned convergence criterion has been met.

Here $\left(n_{1}\right)_{k},\left(n_{3}\right)_{k}$ denote the values $n_{1}$ and $n_{3}$ at the $k$-th iteration, respectively The convergence criterion in Step 5 corresponds to the failure of an increase in $\mathcal{L}(\underline{X} \mid \Theta)$ at a pre assigned tolerance level. A natural starting point for the algorithm to maximize (29) are the MLE's for the STSP distribution (18) given in Van Dorp and Kotz (2002a): 


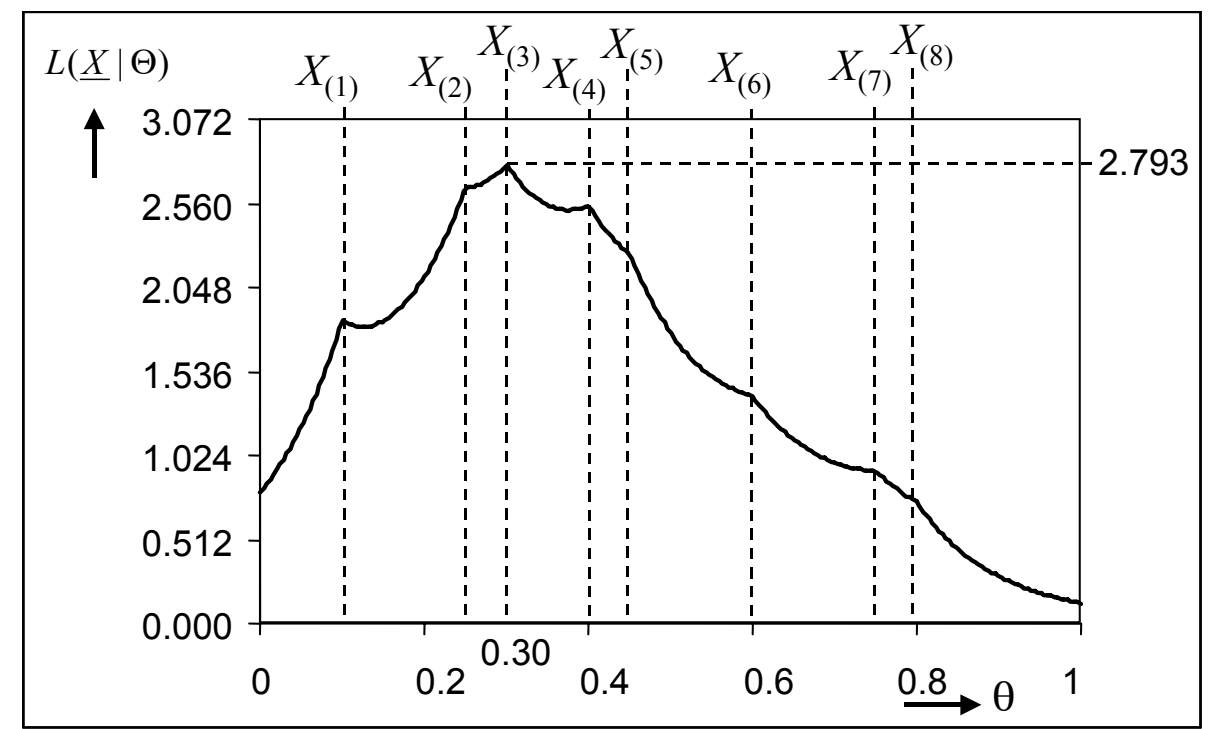

Fig. 6. Example of likelihood $\mathcal{L}(\underline{X} \mid \Theta)$ given by (29) as a function of $\theta$ for the order statistics given by (30) and $n_{1}=n_{3}=2, \alpha=1$.

$$
\left\{\begin{array}{l}
\theta_{0}=X_{(\widehat{r})} \\
\left(n_{1}\right)_{0}=\left(n_{3}\right)_{0}=-\frac{s}{\log M(\widehat{r})} \\
\alpha_{0}=1
\end{array}\right.
$$

where $\widehat{r}=\underset{r \in\{1, \ldots, s\}}{\arg \max } M(r)$ and

$$
M(r)=\prod_{i=1}^{r-1} \frac{X_{(i)}}{X_{(r)}} \prod_{i=r+1}^{s} \frac{\left(1-X_{(i)}\right)}{\left(1-X_{(r)}\right)}
$$

Among the first four steps in a $k$-th iteration described above, Step 4 is the most involved (although straightforward) since it requires maximization of the likelihood (29) over $m+1$ separate intervals $X_{(r)} \leq \theta<X_{(r+1)}, r=0, \ldots, m$, with $X_{(0)} \equiv 0, X_{(m+1)} \equiv 1$. Details regarding these four steps are presented in the Appendix. Finally note that the numerical algorithm described above can easily be modified by omitting Step 3 to provide a maximum likelihood estimation procedure for the GSTSP distribution given by the density (19) which does not involve $\alpha$. 


\section{An example}

We shall illustrate the MLE Procedure for the USTSP distribution utilizing the monthly USA Certificate Deposit rates for the period from 1966-2002. Our aim is to construct a realization of a time series $\nu_{k}, k=0,1,2, \ldots$, from this data, where the $\nu_{k}$ are i.i.d. random variables. This would provide us with an i.i.d. sample for our MLE procedure. To construct such a realization we shall use the by now quite common Auto-Regressive Conditional Heteroscedastic (ARCH) time series model devised by the 2003 Nobel Laureate R.F. Engle in 1982.

The time series of the monthly CD rates is displayed in Figure 7A consisting of 446 data points. Denoting the CD rate after month $k$ by $i_{k}$, our starting point will be one of the simplest financial engineering models for the random behavior of the $\mathrm{CD}$ rate, i.e. the multiplicative model

$$
i_{(k+1) l}=i_{k l} \cdot \epsilon_{k, l}
$$

where $l=1,2, \ldots, 446, k=0, \ldots,\lfloor 446 / l-1\rfloor$ and $\epsilon_{k, l}$ are i.i.d. random variables (see, e.g., Leunberger 1998). Figure 7B depicts the time series of the one-step (i.e. monthly) log differences $(l=1$ in (33))

$$
\operatorname{Ln}\left(\epsilon_{k, 1}\right)=\operatorname{Ln}\left(i_{k+1}\right)-\operatorname{Ln}\left(i_{k}\right)
$$

totaling 445 data points and $i_{0}$ is the monthly CD rate in December of 1965 . Table 2 contains the values of the auto-correlation function

$$
A C F(\lambda, 1)=\operatorname{Corr}\left[\operatorname{Ln}\left(\epsilon_{k+\lambda, 1}\right), \operatorname{Ln}\left(\epsilon_{k, 1}\right)\right]
$$

with lags $\lambda=1, \ldots, 6$ together with the Ljung-Box Q statistics $-L B Q(\lambda)-($ see Ljung and Box 1978) and their p-values for testing the null hypothesis that the auto-correlations for all lags up to lag $\lambda$ equal zero. Tsay (2002) asserts that $\lambda \approx \operatorname{Ln}(446)=6.100$ performs better (in terms of statistical power) than any other values. Table 2 thus contains the values of the $L B Q(\lambda)$ statistic up to $\lambda=6$. From the corresponding p-values it follows immediately that this null hypothesis is rejected for all lags $\lambda=1, \ldots, 6$. 

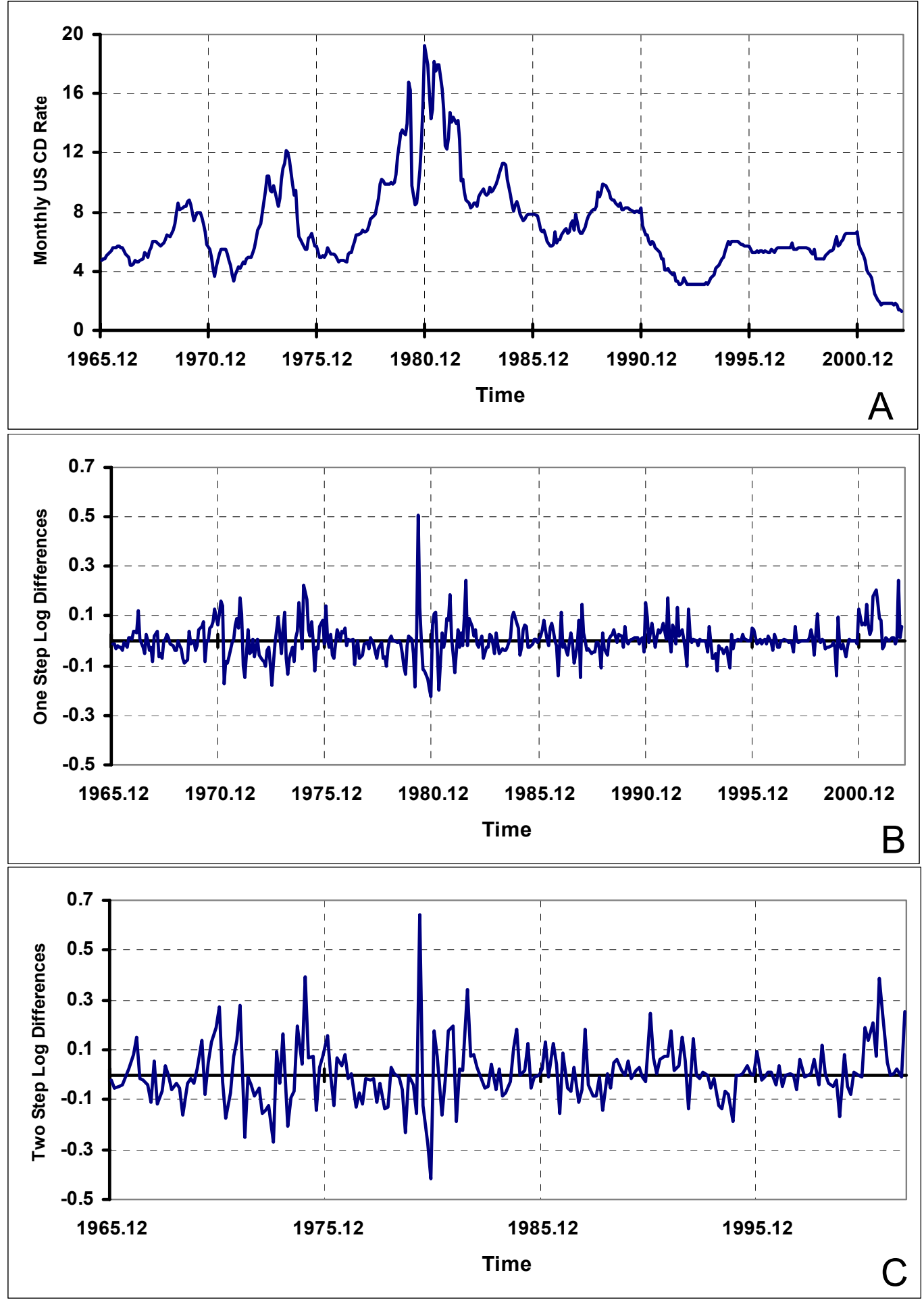

Fig. 7. Monthly US Certificate Deposit Rates from 1966 - 2002 A: Time Series of CD Rates

B: Time Series of One-Step Log Differences; C: Time Series of Two-Step Log Differences. 
Table 2. Auto-correlation Function, Ljung-Box Q Statistic and p-values for one step log differences $\operatorname{Ln}\left(\epsilon_{1, k}\right)$ (cf. (34)) and two-step log differences $\operatorname{Ln}\left(\epsilon_{2, k}\right)$ (cf. (36)) with Lags $1, \ldots, 6$

\begin{tabular}{ccccccc} 
& \multicolumn{3}{c}{ One-Step Log Differences } & \multicolumn{2}{c}{ Two-Step Log Differences } \\
\hline Lag & ACF & LBQ & p-value & ACF & LBQ & p-value \\
\hline 1 & 0.349 & 54.541 & $1.52 \mathrm{E}-13$ & 0.134 & 4.037 & 0.04 \\
2 & 0.092 & 58.321 & $2.17 \mathrm{E}-13$ & 0.040 & 4.404 & 0.11 \\
3 & 0.066 & 60.291 & $5.09 \mathrm{E}-13$ & -0.034 & 4.670 & 0.20 \\
4 & 0.014 & 60.378 & $2.42 \mathrm{E}-12$ & 0.111 & 7.478 & 0.11 \\
5 & 0.007 & 60.401 & $1.00 \mathrm{E}-11$ & 0.060 & 8.305 & 0.14 \\
6 & -0.029 & 60.772 & $3.14 \mathrm{E}-11$ & 0.108 & 10.980 & 0.09 \\
\hline
\end{tabular}

Figure 7C depicts the time series of the two-step (i.e. bi-monthly) log differences ( $l=2$ in (33))

$$
\operatorname{Ln}\left(\epsilon_{k, 2}\right)=\operatorname{Ln}\left(i_{2 k+2}\right)-\operatorname{Ln}\left(i_{2 k}\right)
$$

consisting of $\lfloor 446 / 2-1\rfloor=222$ data points where as before $i_{0}$ is the monthly CD-rate in December 1965. Table 2 also presents the values of the auto-correlation function

$$
A C F(\lambda, 2)=\operatorname{Corr}\left[\operatorname{Ln}\left(\epsilon_{k+\lambda, 2}\right), \operatorname{Ln}\left(\epsilon_{k, 2}\right)\right]
$$

with lags $\lambda=1, \ldots, 6$ together with the corresponding $L B Q(\lambda)$ statistics and their p-values. Note that from the p-values associated with the two step differences $\operatorname{Ln}\left(\epsilon_{k, 2}\right)$ it follows that the null hypothesis (i.e. that auto-correlations for all lags up to lag $\lambda$ equal zero) is accepted for all lags $\lambda=1, \ldots, 6$ (at the significance level of $4 \%$ ). In the case of the 222 data points of the time series (36), the lag $\lambda \approx \operatorname{Ln}(222)=5.403$ performs better in terms of statistical power resulting in a $p$-value of 0.14 in the fifth row of Table 2 . Hence, we may reasonably conclude that the time series $\operatorname{Ln}\left(\epsilon_{k, 2}\right)$ given by (36) is serially uncorrelated.

To test for homoscedasticity ( i.e. a constant variance in the time series (36) and a necessary condition for $\operatorname{Ln}\left(\epsilon_{k, 2}\right)$ given by (36) to be i.i.d.) we shall utilize the systematic framework for volatility modeling provided by the above mentioned ARCH model of Engle (1982). Specifically, an $A R C H(m)$ model assumes that 


$$
a_{k}=\sigma_{k} \nu_{k}, \sigma_{k}^{2}=\alpha_{0}+\alpha_{1} a_{k-1}^{2}+\ldots+\alpha_{m} a_{k-m}^{2}
$$

where $a_{k}$ is serially uncorrelated and $\nu_{k}$ is a sequence of i.i.d. random variables with mean zero and variance 1. For the data in Figure 7.1C, involving $\operatorname{Ln}\left(\epsilon_{k, 2}\right)$, we have

$$
\begin{aligned}
& \overline{\operatorname{Ln}\left(\epsilon_{k, 2}\right)}=\frac{1}{222} \sum_{k=0}^{221} \operatorname{Ln}\left(\epsilon_{k, 2}\right)=0.00561 \\
& s^{2}=\frac{1}{221} \sum_{k=0}^{221}\left(\operatorname{Ln}\left(\epsilon_{k, 2}\right)-\overline{\operatorname{Ln}\left(\epsilon_{k, 2}\right)}\right)=1.419 e-2 .
\end{aligned}
$$

( $s^{2}$ is the sample variance estimator of $\left.\operatorname{Ln}\left(\epsilon_{k, 2}\right), k=1, \ldots, 222\right)$. Hence, the time series

$$
a_{k}=\frac{\operatorname{Ln}\left(\epsilon_{k, 2}\right)}{s}
$$

may be considered a realization of (38) (to avoid cumbersome notation we use the same symbol $\left.a_{k}\right)$. It would seem that using (39) and rescaling $\operatorname{Ln}\left(\epsilon_{k, 2}\right)$ as in (40), one achieves the conditions of a zero mean and variance 1 of $\nu_{k}$ in (38). To further test these conditions, we present in Table 3 the values of its Auto-Correlation Function (ACF) and Partial Auto-correlation Function

\begin{tabular}{|c|c|c|c|c|c|}
\hline Lag & ACF & LBQ & $p$-value & PACF & t-Statistic \\
\hline 1 & 0.096 & 2.067 & 0.15 & 0.096 & 1.428 \\
\hline 2 & 0.098 & 4.239 & 0.12 & 0.090 & 1.336 \\
\hline 3 & 0.231 & 16.395 & 9.41E-04 & 0.218 & 3.248 \\
\hline 4 & 0.098 & 18.581 & $9.50 \mathrm{E}-04$ & 0.058 & 0.866 \\
\hline 5 & 0.001 & 18.581 & $2.30 \mathrm{E}-03$ & -0.050 & -0.740 \\
\hline
\end{tabular}
(PACF) of the time series of $a_{k}^{2}$ up to the lag of 5 (since $\operatorname{Ln}(222)=5.403$ ).

Table 3. Auto-correlation Function (ACF), Ljung-Box Q Statistic, p-values and Partial Auto-Correlation Function (PACF) for $a_{k}^{2}$ (cf. (40)) with Lags $1, \ldots, 5$

As a first check note that the values of the $L B Q$ statistic (and the associated p-values) and the PACF values (in particular those in the third row) in Table 3 suggest that the time series $a_{k}$ given by (40) is heteroscedastic (as opposed to homoscedastic.) From the observation that $a_{k}$ being 
serially uncorrelated (because of (40) and the fact that $\operatorname{Ln}\left(\epsilon_{k, 2}\right)$ are serially uncorrelated) and the PACF values of $a_{k}^{2}$ in Table 3, it follows that $a_{k}$ may be well represented by an $A R C H(3)$ model (see, the third row of Table 3 and Tsay 2002 for a detailed explanation). We thus obtain the following equation for $\sigma_{k}^{2}$

$$
\sigma_{k}^{2}=0.6535+0.06657 a_{k-1}^{2}+0.070194 a_{k-2}^{2}+0.23382 a_{k-3}^{2},
$$

where the parameters $\underline{\alpha}=\left(\alpha_{0}, \ldots, \alpha_{3}\right)$ were estimated using the least squares method (cf. (38)). An alternative test for conditional heteroscedasticity is the so-called Lagrange Multiplier test also due to Engle (1982). This test is equivalent to the usual $F$ statistic for testing $\alpha_{i}=0$, $i=1, \ldots, m$ in the linear regression (38) (see, a basic text on Time Series or Tsay 2002). For our data we have $F=4.9164$ and the p-value of $2.5 e-3$ strongly confirming the earlier conclusion of heteroscedasticity of $a_{k}$ as well as the $A R C H(3)$ setup as given by (38) and (41). Hence, the time series $a_{k}$ is not i.i.d..

However, the set-up for $a_{k}$ in (38) and the values of $\sigma_{k}$ given in (41) suggest that the standardized time series

$$
\nu_{k}=\frac{a_{k}}{\sigma_{k}}, k=3, \ldots, 221,
$$

where $a_{k}$ are given by (40), should be a realization from an i.i.d. time series (by design), which would allows us the use of standard maximum likelihood procedures. Indeed the analysis in Table 4 suggests that the time series $\nu_{k}$ is serially uncorrelated and homoscedastic (possibly with the exception of the $t$-statistic value of 1.423 in the fourth row of Table 4). This could indicate the suitability of an $A R C H(4)$ model for $\nu_{k}$. However, using the linear regression to estimate the time series of $\sigma_{k}^{2}$ in (38) for the sequence $\nu_{k}^{2}, k=3, \ldots, 221$, results in the value for the $\mathrm{F}$ statistic of 0.8048 (to test that $\alpha_{i}=0, i=1, \ldots, 4$ ) with the p-value of 0.52 which strongly supports the homoscedasticity hypothesis of the time series (42). From (36), (40) and (42) it follows that the time series $\nu_{k}$ may be interpreted as that of standardized bi-monthly log- 
differences of US CD rates from 1966-2002 and as above $i_{0}$ is the monthly CD-rate in December 1965.

Table 4. Auto-correlation Function (ACF), Ljung-Box Q Statistic, p-values for $\nu_{k}$, and Partial Auto-Correlation Function (PACF) for $\nu_{k}^{2}$ (cf. (42)) with Lags $1, \ldots, 5$

\begin{tabular}{ccccccc} 
& \multicolumn{3}{c}{$v_{k}$} & & \multicolumn{2}{c}{$v_{k}{ }^{2}$} \\
\hline Lag & & ACF & LBQ & p-value & PACF & t-Statistic \\
\hline 1 & 0.122 & 3.322 & 0.07 & -0.027 & -0.406 \\
2 & 0.099 & 5.490 & 0.06 & -0.033 & -0.484 \\
3 & 0.060 & 6.307 & 0.10 & -0.059 & -0.874 \\
& 4 & -0.004 & 6.310 & 0.18 & 0.096 & 1.423 \\
5 & 0.031 & 6.521 & 0.26 & -0.017 & -0.252 \\
\hline
\end{tabular}

The empirical pdf of the standardized bi-monthly log-differences $\nu_{k}$ is depicted in Figure 1 of Section 1 together with MLE fitted Gaussian (Figure 1A), asymmetric Laplace (Figure 1B), TSP (Figure 1C) and UTSP (Figure 1D) distributions. (For MLE's of Gaussian parameters see a basic text in statistics, e.g., Mood et al., 1974.) Kotz et. al (2002) discuss a MLE procedure for the asymmetric Laplace distribution in some detail. Noting the scale $[-5,5]$ of the graphs in Figure 1, depicting the empirical pdf of $\nu_{k}$ with support $[-2.90,6.97]$, the support of the TSP and UTSP distribution in Figure 1 was set (with an ample safety margin) to be $[-25,25]$. ML estimators for the TSP distribution with a given support are provided in Van Dorp and Kotz (2002b). Before applying the MLE procedures described in Section 4 for the USTSP and GSTSP distributions the data in Figure $7 \mathrm{C}$ was standardized on $[0,1]$ via a linear scale transformation applied to the original support $[-25,25]$. Table 5 contains the ML estimates of the parameters of the pdf's in Figure 1 together with those of a GTSP distribution (19). The parameters $\widehat{\tau} \in$ $[-25,25]$ in Table 5 are obtained by applying the inverse linear scale transformation on the threshold parameter $\widehat{\theta} \in[0,1]$. Figures $8 \mathrm{~A}, 8 \mathrm{~B}, 8 \mathrm{C}$ and $8 \mathrm{D}$ display the empirical cdf of the standardized bi-monthly log-differences of US CD rates together with the ML fitted asymmetric Laplace, TSP, GTSP and UTSP cdf's, respectively. 
Table 5. Maximum Likelihood Estimators for TSP, GTSP and UTSP distributions for the data depicted in Figure 1. The Support for TSP,GTSP and UTSP distributions is $[-25,25]$

\begin{tabular}{lllll}
\hline Gaussian (Figure 1A) & $\widehat{\mu}=7.24 e-3$ & $\widehat{\sigma}=1.095$ & & \\
AS Laplace (Figure 1B) & $\widehat{\theta}=-2.19 e-2$ & $\widehat{\sigma}=1.033$ & $\widehat{\kappa}=9.38 e-1$ & \\
TSP (Figure 1C) & $\widehat{\tau}=-2.07 e-2$ & $\widehat{n}=32.83$ & & \\
GTSP & $\widehat{\tau}=-1.25 e-1$ & $\widehat{n}_{1}=38.24$ & $\widehat{n}_{3}=29.15$ & \\
UTSP (Figure 1D) & $\widehat{\tau}=4.65 e-2$ & $\widehat{n}_{1}=40.29$ & $\widehat{n}_{3}=25.58$ & $\widehat{\alpha}=2.25$ \\
\hline
\end{tabular}
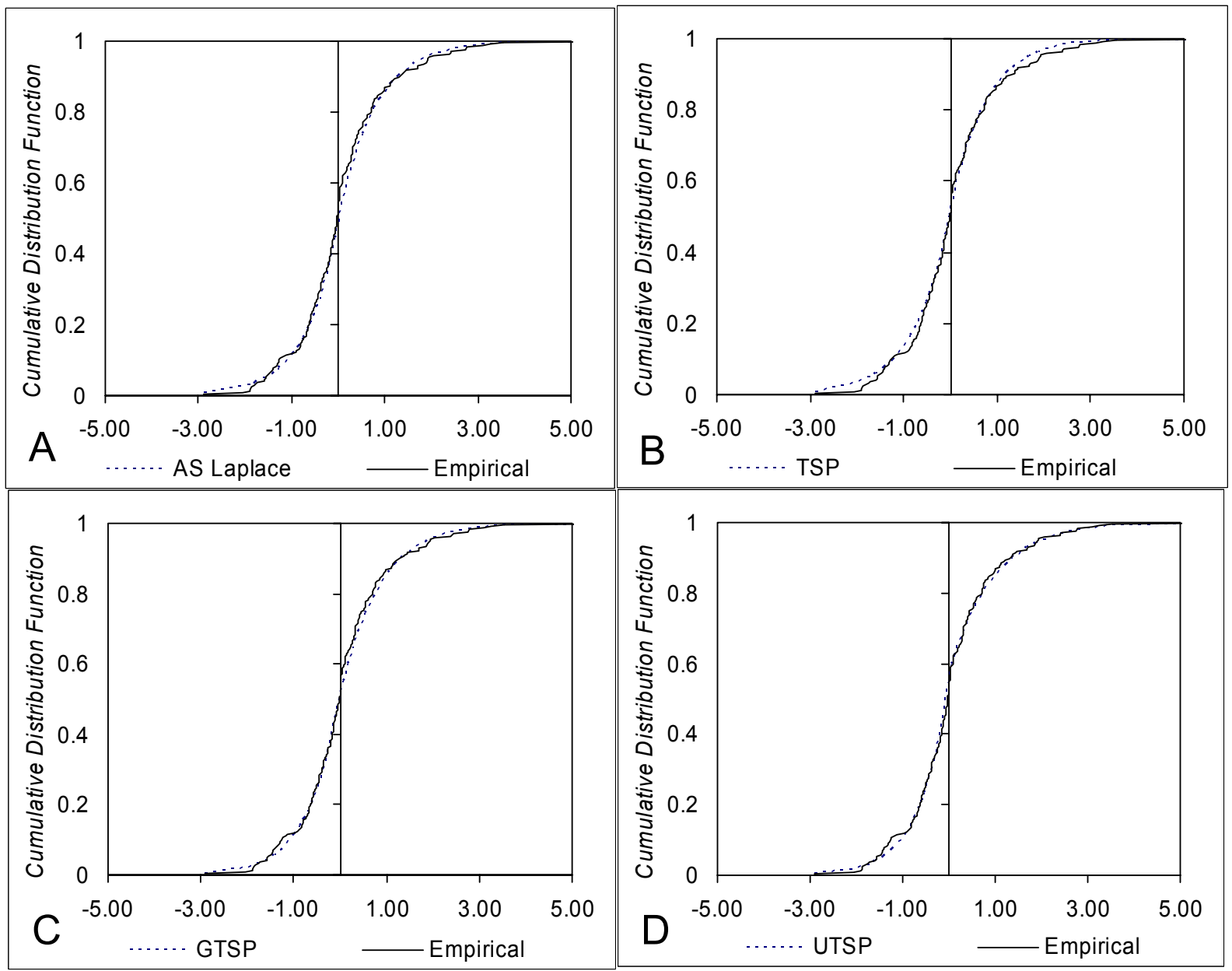

Fig. 8. Empirical CDF of Two-Step Log Differences of Monthly US Certificate Deposit Rates 1966 - 2002 together with MLE fitted distributions: A: Asymmetric Laplace; B: TSP; C: Generalized TSP; D: Uneven TSP (MLE parameters are given in Table 5 above) 
From Figures 1 and 8 we observe (certainly by a careful visual comparison) that the UTSP distribution (Figure 8D) provides a "better" fit to the empirical cdf amongst these five distributions. The GTSP distribution (Figure 8C) and asymmetric Laplace distribution (Figure 8A) seem to perform equally well (and outperform the TSP distribution in Figure 8B). A more formal fit analysis is conducted in Table 6 .

Table 6. Goodness of Fit Analysis of MLE fitted distributions for the 1966-2002 data on standardized bi-monthly Log Differences of Monthly US CD interest rates.

\begin{tabular}{|c|c|c|c|c|c|c|c|c|}
\hline & & & & & \\
\hline & & & & UTSP & GTSP & TSP & AS Laplace & Normal \\
\hline Bin & $\mathrm{LB}_{\mathrm{i}}$ & $\mathrm{UB}_{\mathrm{i}}$ & $\mathrm{O}_{\mathrm{i}}$ & $(\mathrm{Oi}-\mathrm{Ei})^{2} / \mathrm{Ei}$ & $(\mathrm{Oi}-\mathrm{Ei})^{2} / \mathrm{Ei}$ & $(\mathrm{Oi}-\mathrm{Ei})^{2} / \mathrm{Ei}$ & $(\mathrm{Oi}-\mathrm{Ei})^{2} / \mathrm{Ei}$ & $(\mathrm{Oi}-\mathrm{Ei})^{2} / \mathrm{Ei}$ \\
\hline 1 & $<-25$ & -1.482 & 13 & 0.77 & 0.36 & 0.30 & 0.06 & 3.27 \\
\hline 2 & -1.482 & -0.913 & 14 & 0.39 & 0.37 & 0.90 & 0.19 & 3.96 \\
\hline 3 & -0.913 & -0.665 & 14 & 0.01 & 0.05 & 0.04 & 0.27 & 6.3E-05 \\
\hline 4 & -0.665 & -0.521 & 13 & 0.44 & 0.73 & 0.98 & 1.43 & 1.82 \\
\hline 5 & -0.521 & -0.373 & 14 & 1.3E-03 & 0.04 & 0.21 & 0.35 & 1.76 \\
\hline 6 & -0.373 & -0.205 & 14 & 2.05 & 1.30 & 0.56 & 0.52 & 0.49 \\
\hline 7 & -0.205 & -0.125 & 13 & 0.09 & 0.37 & 1.19 & 1.07 & 9.17 \\
\hline 8 & -0.125 & -0.021 & 14 & 0.84 & $2.8 \mathrm{E}-03$ & $1.9 \mathrm{E}-05$ & 0.02 & 5.62 \\
\hline 9 & -0.021 & 0.025 & 14 & 2.84 & 11.54 & 8.78 & 7.91 & 33.63 \\
\hline 10 & 0.025 & 0.108 & 13 & 0.97 & 1.11 & 0.49 & 0.32 & 7.95 \\
\hline 11 & 0.108 & 0.315 & 14 & 0.30 & 2.06 & 2.99 & 3.49 & 0.06 \\
\hline 12 & 0.315 & 0.443 & 14 & 3.54 & 1.16 & 0.79 & 0.56 & 2.73 \\
\hline 13 & 0.443 & 0.713 & 13 & 0.20 & 1.23 & 1.41 & 1.79 & 1.37 \\
\hline 14 & 0.713 & 1.103 & 14 & 0.10 & 0.68 & 0.52 & 0.83 & 3.03 \\
\hline 15 & 1.103 & 1.845 & 14 & 0.37 & 0.67 & 0.17 & 0.50 & 6.60 \\
\hline 16 & 1.845 & $>25$ & 14 & 0.04 & 0.52 & 3.37 & 1.07 & 0.11 \\
\hline \multicolumn{4}{|c|}{ Chi-Squared Statistic } & 12.96 & 22.19 & 22.70 & 20.38 & 81.57 \\
\hline \multicolumn{4}{|c|}{ Degrees of Freedom } & 11 & 12 & 13 & 12 & 13 \\
\hline \multicolumn{4}{|c|}{ P-value } & 0.296 & 0.035 & 0.045 & 0.060 & $<1.00 \mathrm{E}-6$ \\
\hline \multicolumn{4}{|c|}{ Degrees of Freedom (Disc.) } & 9 & 10 & 11 & & \\
\hline \multicolumn{4}{|c|}{ P-value (Discounted) } & 0.164 & 0.014 & 0.019 & & \\
\hline \multicolumn{4}{|c|}{ K-S Statistic } & $3.76 \%$ & $5.58 \%$ & $4.71 \%$ & $7.65 \%$ & $12.75 \%$ \\
\hline \multicolumn{4}{|l|}{ SS } & 0.051 & 0.105 & 0.068 & 0.194 & 1.126 \\
\hline \multicolumn{4}{|c|}{ Log-Likelihood } & -297.04 & -302.83 & -304.45 & -302.55 & -332.39 \\
\hline
\end{tabular}


In Table 6 the Chi-square statistic

$$
\sum_{i=1}^{16} \frac{\left(O_{i}-E_{i}\right)^{2}}{E_{i}}
$$

is calculated utilizing 16 bins $(16 \in[\sqrt{219}, 219 / 5]$ as suggested by Banks et al. 2001). The boundaries of the bins are selected such that the number of observations $O_{i}, i=1, \ldots, 16$, in each Bin $i$ equals 13 or 14, totaling 219 data points. Such a boundary selection procedure partitions the support of the range of observed data in a similar manner as the "equal-probability method of constructing classes" (see, e.g., Stuart et al. 1994) while keeping the bin boundaries of the chi-square statistic the same across the five different distributions depicted in Table 6. The corresponding values $E_{i}, i=1, \ldots, 16$, in (43) for the expected number of observations in Bin $i$ are obtained using

$$
E_{i}=F\left(U B_{i} \mid \widehat{\Theta}\right)-F\left(L B_{i} \mid \widehat{\Theta}\right)
$$

where $F(\cdot \mid \widehat{\Theta})$ is the theoretical cdf, $\widehat{\Theta}$ of the MLE's for the parameters given in Table 5 for each distribution and the bin boundaries $\left(L B_{i}, U B_{i}\right)$ are presented in Table 6 . Note that the Gaussian distribution evidently produces the worst fit with 12 out of the 16 bins contributing a value 1.00 or more to the chi-squared statistic (43). In particular the very high value 33.63 for Bin 9, containing the peak in the empirical pdf, reconfirms the conclusion from Figure 1A that the Gaussian distribution in no way represents such a "peak". While the other fitted distributions (UTSP, GTSP, TSP and asymmetric Laplace) perform much better from bin to bin as compared to the Gaussian distribution, Bin 9 by far contributes the most to the chi-squared statistics regardless of the type of distribution, except for the UTSP case (where it provides the second largest value).

The UTSP distribution yields a better value in terms of the chi-squared statistic not only due to a substantial smaller value in Bin 9, but also because the remaining bins in the UTSP case contribute in total the least to the overall value of the chi-squared statistic compared with the other four distributions. In addition, the UTSP distribution results in the largest p-value of the 
chi-squared hypothesis test taking into account the number of parameters of each distribution to determine the degrees of freedom. This observation also applies to the second pair of $p$-values and degrees of freedom calculated by discounting two additional degrees of freedom for the boundary parameters $[-25,25]$. (It is not quite certain that these two degrees of freedom should be discounted since the boundaries -25 and 25 were not formally estimated from the data but rather obtained by observation).

Table 6 also includes the Kolmogorov-Smirnov Statistic $D$ (see, e.g., Stuart et al. 1994)

$$
D=\operatorname{Max}\left\{D_{i} \mid i=1, \ldots, 219\right\}
$$

where

$$
D_{i}=\operatorname{Max}\left\{\left|\frac{i-1}{219}-F\left(X_{(i)} \mid \widehat{\Theta}\right)\right|,\left|\frac{i}{219}-F\left(X_{(i)} \mid \widehat{\Theta}\right)\right|\right\}
$$

as well as "an intuitive measure of fit"

$$
\sum_{i=1}^{219}\left(\frac{i}{219}-F\left(X_{(i)} \mid \widehat{\Theta}\right)\right)^{2}
$$

denoted by Sum of Squares (SS) (reminiscent of the sum of squares in linear regression analysis) and the log-likelihood

$$
\sum_{i=1}^{219} \operatorname{Ln}\left\{f\left(X_{(i)} \mid \widehat{\Theta}\right)\right\}
$$

where in (46), (47) and (48), $X_{(i)}, i=1, \ldots, 219$, are the order statistics associated with the standardized bi-monthly log-differences $\nu_{k}$ (see, (42)) and the vector $\widehat{\Theta}$ consists of the MLE's of the parameters given in Table 5. Note that the UTSP distribution performs best for all the statistics (43), (45), (47) and (48) amongst the five distributions presented in Table 6. Somewhat unexpectedly, the GTSP and TSP distributions outperform the asymmetric Laplace distribution in terms of the Kolmogorov-Smirnov statistic (45) and the SS (47), but not in terms of the chisquared statistic (43) (and its p-value) and the log-likelihood (48) of the data involving $\nu_{k}$. The K-S statistic and the log-likelihood seem to be much less sensitive to the evident 
inappropriateness of the Gaussian distribution in the situation at hand. In the authors' opinion the behavior of the chi-squared statistic in the indicative Bin 9 in Table 6 (corresponding to the values in the vicinity of the "peak") justifies the conclusion about the suitability of the UTSP distribution for the data under consideration.

\section{Concluding remarks}

This paper is the fifth in the series of our papers on univariate continuous distributions on a bounded domain which attempt to serve as a meaningful alternative to the beta distribution that has been prominently used in practice for many decades (see, Van Dorp and Kotz, 2002a,b and Van Dorp and Kotz 2003a,b). The first (second) paper deals with a two (four) parameter alternative to the beta distribution. The third generalizes the trapezoidal distribution, while the fourth one presents the general structure of continuous two-sided distributions, allowing other generating densities besides the power density. Although the beta function in various forms can be traced to Isaac Newton in the 17th Century (see e.g. Dutka 1981) its popularity and widespread applicability are mainly the result of Karl Pearson's most diligent efforts in constructing his system of empirical distributions at the beginning of the 20th century (especially in the English language literature) and also its use as a prior distribution for the parameter $p$ in binomial models. For application as a prior distribution a reparameterization of the classical beta distribution

$$
\begin{aligned}
& B(x \mid \alpha, \beta)=\frac{(b-a)^{1-(\alpha+\beta)}}{\mathbb{B}(\alpha, \beta)} \int_{0}^{x}(u-a)^{\alpha-1}(b-u)^{\beta-1} d u, \\
& \alpha>0, \beta>0, \mathbb{B}(\alpha, \beta)=\frac{\Gamma(\alpha) \Gamma(\beta)}{\Gamma(\alpha+\beta)}
\end{aligned}
$$

exists that allows for a transparent interpretation of its parameters (see, e.g. Clemen and Reilly 2001). Unfortunately however, the parameters $\alpha$ and $\beta$ in the four parameter beta distribution (49) seem to lack meaningful physical interpretation and in addition present computational difficulties in maximum likelihood estimation (see, e.g., Mielke 1975). This was one of the reasons that prompted us to devise an alternative family which is based on possibly more sound 
physical and statistical motivations. The general UTSP family described in this paper - to the best of our knowledge - cannot be constructed via the beta distribution. To summarize: a new UTSP distribution on a bounded interval with meaningful parameters (which is suitable for description of phenomena in various sciences and engineering possessing a jump discontinuity) and a straightforward maximum likelihood estimation procedure for its parameters have been developed in this paper.

\section{Appendix: The maximum likelihood algorithm}

Consider the likelihood given by (29). Below we shall provide some details regarding the first four steps in the $k$-th iteration presented in Section 4 and the MLE procedure maximizing (29). (Compare this description with the procedure presented in Van Dorp and Kotz (2002a) for a simpler case.)

Step 1 : Maximizing over the left hand side power parameter $n_{1}$

We shall separately consider the two cases $A: X_{1} \leq \ldots \leq X_{(r)} \leq \theta<X_{(r+1)}$ and $B$ : $\theta<X_{(1)}$.

Case 1.A : Assuming $X_{1} \leq \ldots \leq X_{(r)} \leq \theta<X_{(r+1)}$ and introducing the notation

$$
0<\mathcal{A}=\prod_{i=1}^{r} \frac{X_{(i)}}{\theta}<1 ; \mathcal{B}=(1-\theta)>0 ; \mathcal{C}=\alpha \theta n_{3}>0
$$

we may rewrite the likelihood function (29) in the form

$$
\mathcal{L}\left(\underline{X} \mid n_{1}\right) \propto \mathcal{A}^{n_{1}-1}\left\{\frac{n_{1}}{\mathcal{C}+\mathcal{B} n_{1}}\right\}^{m} .
$$

From (A.1) and (A.2) it follows that $\mathcal{L}(\underline{X} \mid 0)=0, \mathcal{L}\left(\underline{X} \mid n_{1}\right)>0$ for $n_{1}>0$ and $n_{1} \stackrel{\lim }{\rightarrow} \mathcal{L}\left(\underline{X} \mid n_{1}\right)=0$ for $0<\mathcal{A}<1$. Hence $\mathcal{L}\left(\underline{X} \mid n_{1}\right)$ attains its maximum at some stationary point $n_{1}^{*}>0$. Instead of maximizing $\mathcal{L}\left(\underline{X} \mid n_{1}\right)$ we equivalently maximize its logarithm

$$
\left(n_{1}-1\right) \operatorname{Ln}(\mathcal{A})+m \operatorname{Ln}\left(n_{1}\right)-m \operatorname{Ln}\left(\mathcal{C}+\mathcal{B} n_{3}\right) .
$$

Setting $d \operatorname{Ln}\left(\mathcal{L}\left(\underline{X} \mid n_{1}\right)\right) / d n_{1}=0$, yields 


$$
\mathcal{B} \operatorname{Ln}(\mathcal{A})\left(n_{1}\right)^{2}+\mathcal{C} \operatorname{Ln}(\mathcal{A}) n_{1}+m \mathcal{C}=0
$$

Since, the quadratic equation (A.4) has at most two real valued solutions, we conclude, noting that $\mathcal{L}(\underline{X} \mid 0)=0, \mathcal{L}\left(\underline{X} \mid n_{1}\right)>0$ for $n_{1}>0$ and ${ }_{n_{1}} \lim _{\rightarrow} \infty \mathcal{L}\left(\underline{X} \mid n_{1}\right)=0$, that $\mathcal{L}\left(\underline{X} \mid n_{1}\right)$ has a unique stationary point $n_{1}^{*}>0$, which may be obtained from (A.4) utilizing (A.1).

Case 1.B : Assuming $\theta<X_{(1)}$ and rewriting (29) using (A.1) we have

$$
\mathcal{L}\left(\underline{X} \mid n_{1}\right) \propto\left\{\frac{n_{1}}{\mathcal{C}+\mathcal{B} n_{1}}\right\}^{m} .
$$

Note that, the term $\mathcal{A}^{n_{1}-1}$ in (A.2) is not included in (A.5). From (A.5) it follows, taking (A.1) into account, that $\mathcal{L}(\underline{X} \mid 0)=0, \mathcal{L}\left(\underline{X} \mid n_{1}\right)>0$ for $n_{1}>0,{ }_{n}$ lim $\rightarrow \infty$

$$
\left.d \mathcal{L}\left(\underline{X} \mid n_{1}\right)\right) / d n_{1}=m\left\{\frac{n_{1}}{\mathcal{C}+\mathcal{B} n_{1}}\right\}^{m-1} \frac{\mathcal{C}}{\left(\mathcal{C}+\mathcal{B} n_{1}\right)^{2}}>0
$$

for $n_{1}>0$. Hence, Case $B$ does not yield a maximum solution for $\mathcal{L}\left(\underline{X} \mid n_{1}\right)$. However, one may rule this situation out by using the starting solution given by (31) (corresponding to the STSP case) or a histogram of the data of a unimodal form which contains observations on both sides of the mode. If nevertheless this case does occur, no solution can be found and the algorithm terminates.

Step 2 : Maximizing over the right hand side power parameter $n_{3}$ As be before, we shall separately consider the two cases $A: X_{(r)} \leq \theta<X_{(r+1)}<\ldots<X_{(m)}$ and $B: \theta \geq X_{(m)}$.

Case 2.A : Assuming $X_{(r)} \leq \theta<X_{(r+1)}<\ldots<X_{(m)}$ and introducing the notation

$$
0<\mathcal{D}=\prod_{i=r+1}^{m} \frac{1-X_{(i)}}{1-\theta}<1 ; \mathcal{E}=\alpha \theta>0 ; \mathcal{F}=(1-\theta) n_{1}>0
$$

we rewrite (29) in the form

$$
\mathcal{L}\left(\underline{X} \mid n_{3}\right) \propto \mathcal{D}^{n_{3}-1}\left\{\frac{n_{3}}{\mathcal{F}+\mathcal{E} n_{3}}\right\}^{m}
$$


Analogously to (A.2), by taking the derivative of $\operatorname{Ln}\left\{\mathcal{L}\left(\underline{X} \mid n_{3}\right)\right\}$, we obtain the unique solution $n_{3}^{*}>0$, maximizing $\mathcal{L}\left(\underline{X} \mid n_{3}\right)$, by solving the quadratic equation

$$
\mathcal{E} \operatorname{Ln}(\mathcal{D})\left(n_{3}\right)^{2}+\mathcal{F} \operatorname{Ln}(\mathcal{D}) n_{3}+m \mathcal{F}=0 .
$$

As in Step 1 no solution can be found here for Case 2.B and the algorithm terminates.

Step 3 : Maximizing over the jump parameter $\alpha$

Introducing the notation

$$
\mathcal{G}=\theta n_{3}>0 ; \mathcal{H}=(1-\theta) n_{1}>0
$$

we now rewrite (29) in the form

$$
\mathcal{L}(\underline{X} \mid \alpha) \propto \frac{\alpha^{r}}{\{\mathcal{G} \alpha+\mathcal{H}\}^{m}}
$$

As above, instead of maximizing $\mathcal{L}(\underline{X} \mid \alpha)$, we equivalently maximize its logarithm

$$
r \operatorname{Ln}(\alpha)-m \operatorname{Ln}\{\mathcal{G} \alpha+\mathcal{H}\}
$$

Setting $d \operatorname{Ln}\{\mathcal{L}(\underline{X} \mid \alpha)\} / d \alpha=0$, yields

$$
(r-m) \mathcal{G} \alpha+r \mathcal{H}=0 .
$$

From $\operatorname{Sign}[d \operatorname{Ln}\{\mathcal{L}(\underline{X} \mid \alpha)\} / d \alpha]=\operatorname{Sign}\{(r-m) \mathcal{G} \alpha+r \mathcal{H}\}$ it follows from (A.12) and deciphering notation (A.10) that

$$
\alpha^{*}=\frac{r \mathcal{H}}{(m-r) \mathcal{G}}=\frac{r \theta n_{3}}{(m-r)(1-\theta) n_{1}}>0
$$

maximizes $\mathcal{L}(\underline{X} \mid \alpha)$ in (A.11). (Note that an increase in $\theta$ increases the value of the jump parameter $\alpha$ as stated indicated in Section 3).

Step 4 : Maximizing over the threshold parameter $\theta$ for the case $n_{1} \geq 1$ and $n_{3} \geq 1$. Instead of maximizing $\mathcal{L}(\underline{X} \mid \Theta)$ given by (29) as a function of $\theta$, we minimize its reciprocal

$$
\frac{1}{\mathcal{L}(\underline{X} \mid \theta)} \propto\left(\left(\alpha n_{3}-n_{1}\right) \theta+n_{1}\right)^{m} \theta^{\left(n_{1}-1\right) r}(1-\theta)^{\left(n_{3}-1\right)(m-r)}
$$

over the set 


$$
X_{(r)} \leq \theta<X_{(r+1)}, 0 \leq r \leq m,
$$

with $X_{(0)} \equiv 0, X_{(m+1)} \equiv 1$. This is the basic quantity which will be manipulated in all the cases of Step 4. The difficulty in minimizing (A.15) over the set of values (A.16) is that $(m+1)$ separate disjoint bounded intervals ought to be considered, each of which could potentially contain the solution minimizing (A.15). To minimize the reciprocal of the likelihood (A.15), we shall separately consider the three cases

$$
\begin{gathered}
\text { Case 4.A: } X_{(r)} \leq \theta<X_{(r+1)}, 1 \leq r \leq m-1, \\
\text { Case } 4 . B: 0 \leq \theta<X_{(1)}, r=0 ; \\
\text { Case } 4 . C: X_{(m)} \leq \theta \leq 1, r=m .
\end{gathered}
$$

Each of these cases yields a potential solution for $\theta: \theta^{A}, \theta^{B}$ and $\theta^{C}$, respectively. Next, we evaluate (A.15) at these three values $\theta^{A}, \theta^{B}$ and $\theta^{C}$ and select the one that yields the lowest value of $\mathcal{L}^{-1}(\underline{X} \mid \theta)$ given in (A.15).

Case 4.A: $X_{(r)} \leq \theta<X_{(r+1)}, 1 \leq r \leq m-1$ : When minimizing (A.15) over $X_{(r)} \leq \theta<X_{(r+1)}$ for a specific value of $r$, the minimum is attained at either $X_{(r)}$ or $X_{(r+1)}$ or at a stationary point $\theta^{*}$ such that $X_{(r)} \leq \theta^{*}<X_{(r+1)}$. Introducing the notation

$$
\mathcal{K}=\left(n_{1}-1\right) r ; \quad \mathcal{M}=\left(n_{3}-1\right)(m-r),
$$

the function

$$
g_{A}(\theta)=\theta^{\mathcal{K}}(1-\theta)^{\mathcal{M}}
$$

and setting $d \mathcal{L}^{-1}(\underline{X} \mid \theta) / d \theta=0$, we have

$$
\begin{gathered}
\left(\left(\alpha n_{3}-n_{1}\right) \theta+n_{1}\right)^{m-1}\left[m g_{A}(\theta)+\left(\left(\alpha n_{3}-n_{1}\right) \theta+n_{1}\right) g_{A}^{\prime}(\theta)\right]=0 \Leftrightarrow \\
\text { either } \theta=\frac{n_{1}}{n_{1}-\alpha n_{3}} \text { or } \frac{\left(\left(\alpha n_{3}-n_{1}\right) \theta+n_{1}\right)}{m}=\frac{-g_{A}(\theta)}{g_{A}^{\prime}(\theta)} .
\end{gathered}
$$


Since for all values of $n_{1}, n_{3}, \alpha>0$, the first solution in (A.22) is less than 0 or larger than 1 and only the second one can provide a solution $X_{(r)} \leq \theta^{*}<X_{(r+1)}$. Hence, we have using (A.20) that

$$
\begin{gathered}
\frac{\left(\left(\alpha n_{3}-n_{1}\right) \theta+n_{1}\right)}{m}=\frac{\theta(1-\theta)}{\mathcal{K}-(\mathcal{K}+\mathcal{M}) \theta} \Leftrightarrow \\
-\left\{\left(\alpha n_{3}-n_{1}\right)(\mathcal{K}+\mathcal{M})+m\right\} \theta^{2}+\left\{m-n_{1}(\mathcal{K}+\mathcal{M})+\left(\alpha n_{3}-n_{1}\right) \mathcal{K}\right\} \theta+n_{1} \mathcal{K}=0
\end{gathered}
$$

Expression (A.23) is a simple quadratic equation which possesses at most two solutions. Hence, to minimize (A.15) over $X_{(r)} \leq \theta<X_{(r+1)}$, for a specific value of $r$, we evaluate the reciprocal of the likelihood (A.15) at $\theta^{*}=X_{(r)}, \theta^{*}=X_{(r+1)}$ and the solutions of the quadratic equation (A.23), provided these solutions $\theta^{*}$ satisfy $X_{(r)} \leq \theta^{*}<X_{(r+1)}$, and set $\theta_{(r)}$ to that value of $\theta^{*}$ that yields the minimum of $\mathcal{L}^{-1}(\underline{X} \mid \theta)$ given in (A.15) amongst these two to four possibilities. Next, we evaluate (A.15) at $\theta_{(r)}$ for $r=1, \ldots, m-1$ and set $\theta^{A}$ to be the value of $\theta_{(r)}$ that yields the minimum of (A.15) over the set of values defined by (A.17).

Case $4 . B: 0 \leq \theta<X_{(1)}, r=0$ : When minimizing (A.15) over $0 \leq \theta<X_{(1)}$, the minimum is attained at either 0 or $X_{(1)}$ or at a stationary point $\theta^{*}$ such that $0 \leq \theta^{*}<X_{(1)}$. Analogously to Case 4 . A additional solutions for $0 \leq \theta^{*}<X_{(1)}$ may be found by solving

$$
\frac{\left(\left(\alpha n_{3}-n_{1}\right) \theta+n_{1}\right)}{m}=\frac{-g_{B}(\theta)}{g_{B}^{\prime}(\theta)}
$$

where

$$
g_{B}(\theta)=(1-\theta)^{\mathcal{M}}
$$

and $\mathcal{M}$ is given by (A.20) for $r=0$. Hence, we have

$$
\theta^{*}=\frac{1+n_{1}\left(n_{3}-1\right)}{1+\left(n_{1}-\alpha n_{3}\right)\left(n_{3}-1\right)} .
$$

In summary, to minimize (A.15) over $0 \leq \theta<X_{(1)}, r=0$ we evaluate (A.15) at $\theta^{*}=0$, $\theta^{*}=X_{(1)}$ and $\theta^{*}$ given by equation (A.26) (provided that this solution $\theta^{*}$ satisfies 
$\left.0 \leq \theta^{*}<X_{(1)}\right)$ and then set $\theta^{B}$ to be the value of $\theta^{*}$ which yields the minimum of (A.15) amongst these three possibilities.

Case 4.C : $X_{(m)} \leq \theta \leq 1, r=m$ : When minimizing (A.15) over $X_{(m)} \leq \theta \leq 1$ the

minimum is attained at either $X_{(m)}$ or 1 or at a stationary point $\theta^{*}$ such that $X_{(m)} \leq \theta \leq 1$. Analogously to Case $A$ additional solutions $X_{(m)} \leq \theta^{*} \leq 1$ may be found by solving

$$
\frac{\left(\left(\alpha n_{3}-n_{1}\right) \theta+n_{1}\right)}{m}=\frac{-g_{C}(\theta)}{g_{C}^{\prime}(\theta)}
$$

where

$$
g_{C}(\theta)=\theta^{\mathcal{K}}
$$

and $\mathcal{K}$ is given in (A.20) by setting now $r=m$. Hence, we have

$$
\theta^{*}=\frac{n_{1}\left(n_{1}-1\right)}{\left(n_{1}-\alpha n_{3}\right)\left(n_{1}-1\right)-1} .
$$

(Compare with (A.26)). Thus, to minimize (A.15) over $X_{(m)} \leq \theta \leq 1, r=m$, we evaluate (A.15) at $\theta^{*}=X_{(m)}, \theta^{*}=1$ and $\theta^{*}$ given by equation (A.29) (provided that this solution $\theta^{*}$ satisfies $X_{(m)} \leq \theta \leq 1$ ) and set $\theta^{C}$ to be the value of $\theta^{*}$ which yields the minimum of (A.29) amongst these three possibilities. 


\section{References}

Aigner DJ, Amemiya T, and Poirier, D J (1976) On the Estimation of Production Frontiers:

Maximum Likelihood Estimation of the Parameters of a Discontinuous Density Function.

International Economic Review 17 (2): 377-396

Banks J, Carson J S, Nelson B L, and Nicol, D M (2001) Discrete-Event System Simulation (3rd ed.). Prentice-Hall, Upper Saddle River NJ, pp. 343-345

Barnard G A (1989) Sophisticated Theory and Practice in Quality Improvement. Philosophical Transactions of The Royal Society A 327: 581-589

Bowlus A, Neumann G, Kiefer A (2001) Equilibrium Search Models and the Transition from School to Work. International Economic Review 42 (2): 317-343

Brown S L (1999) An SAB Report: Estimating Uncertainties in Radiogenic Cancer Risk. Science and Advisory Board, United States Environmental Protection Agency, EPA-SAB-RAC-99-008, Washington D.C.

Chen S J, Hwang CL (1992) Fuzzy Multiple Attribute Decision-Making: Methods and Applications. Springer-Verlag, Berlin NY.

Chernozhukov V, Hong H (2002) Likelihood Inference in a Class of Non regular Econometric Models. MIT Department Working Paper No. 02-05

Clemen R T, Reilly T (2001) Making Hard Decisions with Decision Tools. Duxbury, Pacific Grove CA 
Donald S G, Paarsch H J (1996) Identification, Estimation, and Testing in Parametric Empirical Models of Auctions within Independent Private Values Paradigm. Econometric Theory 12: 517567

Dutka J (1981) The Incomplete Beta Function - A Historical Profile. Archive for History of Exact Science 24: 11-29

Engle R F (1982) Autoregressive Conditional Heteroscedasticity with Estimates of the Variance of United Kingdom Inflations. Econometrica 50: 987-1007

Escher M C (1989) Escher on Escher. Harry N. Abrams, New York NY

Flehinger B J, Kimmel M (1987) The Natural History of Lung Cancer in Periodically Screened Population. Biometrics 43: 127-144

Fechner G T (1897) Kollectivemasslehre. Wilhelm Englemann, Leipzig

Kanefsky M, Thomas J B (1965) On Polarity Detection Schemes with Non-Gaussian Inputs. Journal of the Franklin Institute 280 (2): 120-138

Klein G E (1993) The Sensitivity of Cash-Flow Analysis to the Choice of Statistical model for Interest Rate Changes (with discussions). Transactions of the Society of Actuaries XLV: 79-186

Kozubowski T J, Podgórski K (1999) A Class of Asymmetric Distributions. Actuarial Research Clearing House 1: 113-134 
Kotz S, Kozubowski T J, Podgórski K (2002) Maximum Likelihood Estimation of Asymmetric Laplace Parameters. Annals of the Institute of Statistical Mathematics 54 (4): 816-826

Leunberger D G (1998) Investment Science. Oxford University Press, New York NY

Ljung G M and Box G E P (1978) On a Measure of Lack of Fit in Time Series Models. Biometrika 65: 297-304

Mielke P W Jr (1975) Convenient Beta Distribution Likelihood Techniques for Describing and Comparing Meteorological Data. Journal of Applied Meteorology 14 (6): 985-990

Mood A, Graybill A F, Boes D C (1974) Introduction to the Theory of Statistics (3rd ed.). McGraw-Hill, Singapore

Pouliquen L Y (1970) Risk Analysis in Project Appraisal. World Bank Staff Occasional Papers, 1, John Hopkins University Press, Baltimore MD

Powell M R, Wilson J D (1997) Risk Assessment for National Natural Resource Conservation Programs. Discussion Paper 97-49. Resources for the Future, Washington, DC.

Stuart A S, Ord K, Arnold S (1999) Kendall's Advanced Theory of Statistics, Volume 2A: Classical Inference and the Linear Model (6th ed.). Edward Arnold, London

Tsay R S (2002) Analysis of Financial Time Series. Wiley, New York NY

Van Dorp J R, Kotz S (2002a) The Standard Two Sided Power Distribution and its Properties: with Applications in Financial Engineering. The American Statistician 56 (2): 90-99 
(2002b) A Novel Extension of the Triangular Distribution and its Parameter Estimation.

Journal of Royal Statistical Society, Series D, The Statistician 51 (1): 63-79

(2003a) Generalized Trapezoidal Distributions. Metrika 58 (1): 85-97

(2003b) Generalizations of Two sided Power Distributions and their Convolution.

Communications in Statistics: Theory and Methods 32 (9): 1703 - 1723

Weyl H (1952) Symmetry. Princeton University Press, Princeton NJ

Zabell S L (1988) Symmetry and its discontents. In: B. Skyrms \& W.L. Harper (eds) Causation, chance and credence, 1. Kluwer, Dordrecht pp 155-190 